\title{
Perspectives rituelles dans les Plaines et sur la Côte Nord-Ouest
}

Ritual perspectives in the Plains and on the North West Coast

Perspectivas rituales en las Grandes Llanuras y en la Costa Noroeste

\section{Klaus Hamberger}

\section{(2) OpenEdition \\ Journals}

Édition électronique

URL : https://journals.openedition.org/jsa/14739

DOI : 10.4000/jsa. 14739

ISSN : $1957-7842$

\section{Éditeur}

Société des américanistes

Édition imprimée

Date de publication : 31 octobre 2016

Pagination : 43-78

ISSN : 0037-9174

Référence électronique

Klaus Hamberger, "Perspectives rituelles dans les Plaines et sur la Côte Nord-Ouest », Journal de la Société des américanistes [En ligne], 102-1 | 2016, mis en ligne le 07 décembre 2016, consulté le 03 septembre 2022. URL : http://journals.openedition.org/jsa/14739; DOI : https://doi.org/10.4000/jsa. 14739

Tous droits réservés 


\title{
Perspectives rituelles dans les Plaines et sur la Côte Nord-Ouest
}

\author{
Klaus HAMBERGER *
}

\begin{abstract}
À travers une analyse transformationnelle des rites saisonniers historiques des Plaines et de la Côte Nord-Ouest, l'article visera à explorer la logique dynamique de l'espace rituel en Amérique du Nord. Nous tenterons de montrer que l'architecture de la « danse du soleil » et de la « cérémonie d'hiver » servait non seulement à créer un espace virtuel, axé sur l'antagonisme entre la position de prédateur en haut et de gibier en bas, mais aussi à inverser ces positions en transformant les perspectives. Cette transformation de perspective, au cœur de la dynamique interne de chacun des rituels, constitue en même temps une clé pour comprendre les liens logiques qu'ils entretiennent entre eux: ils construisent le même espace de deux points de vue opposés. Dans les Plaines, il s'agissait de transférer aux humains la puissance des prédateurs surnaturels; sur la Côte Nord-Ouest, il s'agissait de retransformer des prédateurs surnaturels en êtres humains. Dans le premier cas, la transformation de perspective était orientée de bas en haut; dans le second, de haut en bas. Ces différentes orientations sont corrélées avec les saisons où se déroulaient respectivement ces rituels : l'été, période de mobilité où l'on se rendait collectivement chez les autres (gibier, ennemis, esprits), ou l'hiver, période d'immobilité où l'on recevait les autres (non-humains) chez soi. Ces différents modes relationnels étant sexués, la dynamique de l'espace rituel s'inscrit dans la variation saisonnière des relations de genre qui caractérisait la morphologie sociale nord-amérindienne. [Mots-clés : Plaines, Côte Nord-Ouest, danse du soleil, cérémonie d'hiver, espace, genre, rituel.]
\end{abstract}

Ritual perspectives in the Plains and on the North West Coast. Via a transformational analysis of the historical Plains and Northwest Coast seasonal rituals, the article seeks to explore the dynamic logic of North Amerindian social space. We shall endeavor to show that the architecture of the « Sun Dance » and the "Winter Ceremonial » not only served to create a virtual space centered on the polarity between the positions of predator (above) and prey (below), but also to invert these positions by a transformation of perspectives. This perspectival transformation, at the heart of each of the rituals, constitutes also a key to the understanding of the logical connection between them: they construct the same space from two opposite

* École des hautes études en sciences sociales, Laboratoire d'anthropologie sociale [klaus. hamberger@ehess.fr]. 
points of view. In the Plains, the ritual aim was to transfer the power of supernatural predators to humans; on the Northwest Coast, to re-transform supernatural predators into human beings. In the first case, the transformation of perspectives was oriented upwards; in the second, downwards. These different orientations are correlated with the seasons in which the rituals took place: summer, a period of mobility where one visited « others » (prey animals, enemies, spirits); or winter, a period of immobility where the (non-human) « others » were received at home. As these different modes of relationship are gendered, the dynamics of ritual space partakes of the seasonal variation of gender relations that characterized native North American social morphology. [Key words: Plains, Northwest Coast, sun dance, winter ceremonial, space, gender, ritual.]

Perspectivas rituales en las Grandes Llanuras y en la Costa Noroeste. A través de un análisis transformacional de los rituales estacionales históricos en las Grandes Llanuras y en la Costa Noroeste, el artículo investiga la lógica dinámica del espacio ritual en América del Norte. Intentaremos demostrar que la arquitectura de la « danza del sol » y de las « ceremonias de invierno » servía tanto para crear un espacio ritual centrado en el antagonismo entre la posición del depredador arriba y la de la presa abajo, como para invertir las posiciones, transformando las perspectivas. Este cambio de perspectiva mismo, que está en el centro de la dinámica interna de cada uno de los rituales, constituye mientras tanto al mismo tiempo una clave para entender los vínculos lógicos entre ellos: dichos ritos construyen un mismo espacio ritual desde dos puntos de vista opuestos. Si en las Grandes Llanuras, se trataba de transferir a los seres humanos la potencia de los depredadores sobrenaturales; en la Costa Noroeste, era cuestión, al revés, de volver a transformar a los depredadores sobrenaturales en seres humanos. Mientras que en el primer caso el cambio de perspectiva se orientaba de abajo hacia arriba, en el segundo, era de arriba hacia abajo. Estas diferentes orientaciones se correlacionan directamente con las estaciones del año en las que ambos rituales se llevaban a cabo: el verano correspondía a un periodo de movilidad durante el cual se visitaba a los otros (sean cazas, enemigos, espíritus) mientras que durante el inverno uno se quedaba en casa preocupándose de acoger a los otros (no humanos). Estos modos relacionales son además sexuados. Así, la dinámica del espacio ritual se inscribe también en la variación estacional de las relaciones de género que caracterizaba la morfología social de los amerindios del Norte. [Palabras clave: Grandes Llanuras, Costa Noroeste, danza del sol, ceremonias de invierno, espacio, género, ritual.]

\section{Introduction}

L'Amérique du Nord a constitué un terrain particulièrement fécond pour l'évolution de la méthode transformationnelle ${ }^{1}$. C'est en grande partie à travers

1. Ce texte est issu d'un séminaire qui s'est tenu à l'EHESS en 2011-2012. Je suis redevable à Isabelle Daillant, Emmanuel Désveaux, Vincent Hirtzel, Michael Houseman, Dimitri Karadimas, Marie Mauzé, Patrick Pérez, Perig Pitrou, Cédric Yvinec et aux lecteurs 
les matériaux des Plaines que Lévi-Strauss s'est attaqué, dans le $3^{\mathrm{e}}$ volume des Mythologiques, à l'étude transformationnelle des relations (voir LéviStrauss 1968, p. 388). Les études de Rosman et Rubel (1971), Reid (1981) et Désveaux (2007 [2000], p. 264-268; 2001, p. 101 et suiv., p. 269 et suiv.) sur la fête d'hiver et la danse du soleil comptent parmi les rares exemples d'analyses transformationnelles du rituel ${ }^{2}$. Emmanuel Désveaux (2001) est allé plus loin encore, en appliquant la méthode transformationnelle à l'ensemble des matériaux ethnographiques nord-américains.

Les sociétés amérindiennes qui habitaient au XIX ${ }^{\mathrm{e}}$ siècle la région située entre les Plaines et l'Arctique se prêtent en effet à ce genre d'analyses. Elles se caractérisaient par une forte variation saisonnière qui affectait toutes les dimensions de leur morphologie sociale, et elles pratiquaient des rituels saisonniers élaborés qui soumettaient les participants à d'importantes expériences de métamorphose. La notion de transformation s'applique ici non seulement aux rapports entre sociétés différentes, mais à la dynamique interne de chacune. Il n'est donc pas surprenant que la méthode transformationnelle ne se soit rarement montrée aussi productive que dans le contexte nord-américain.

Cette foisonnante richesse a paradoxalement eu pour effet de jeter un doute sur son bien-fondé. Dans un réseau sémantique aussi dense, ne finira-t-on pas toujours par construire tout groupe de transformations voulu? À ces doutes méthodologiques se joignaient des objections d'ordre épistémologique. Quel modèle de l'esprit humain autorisait en effet à penser que l'opération de « transformation » ait une réalité cognitive au-delà des desseins de l'anthropologue? Ces critiques n'étaient pas infondées, et des baguettes magiques telles que la « formule canonique » n'ont pas aidé à les invalider. En fait, il était difficile de les contrer tant que l'analyse transformationnelle tenait au paradigme classique de la langue, plutôt que de se tourner résolument vers l'autre paradigme auquel elle avait pourtant emprunté le concept de la transformation : celui de l'espace.

En se concentrant sur les systèmes de coordonnées par lesquels les sociétés organisent leur espace social, l'analyse transformationnelle se donne un cadre plus transparent, basé sur un nombre réduit de relations. Elle donne au concept de « transformation » le sens palpable d'un changement de perspective. Enfin, au lieu de projeter les dispositifs de transformation à l'intérieur de l'esprit humain, elle les trouve dans la réalité observable des architectures domestiques et rituelles.

C'est dans cette optique que nous nous proposons d'étudier la dynamique topologique des sociétés des Plaines et de la Côte Nord-Ouest au prisme de leurs

anonymes du JSA pour leurs commentaires sur des versions antérieures, ainsi qu'à Marie Mauzé qui a généreusement partagé ses matériaux ethnographiques.

2. Lévi-Strauss lui-même ne s'est intéressé aux rituels de la région que dans le contexte de la mythologie associée (1968, p. 161-266; 1971, p. 452 et suiv., p. 532; 1973 [1971]; 1979, p. 63 et suiv.). 
rituels saisonniers. Tâche à la fois facilitée et compliquée par l'état très avancé des recherches dans ce domaine - de l'analyse lévi-straussienne de la mythologie de la danse du soleil au modèle général du lien entre sexe, société et saison proposé par Désveaux. Si notre lecture des sources se distingue des leurs, ce n'est pas tant par rapport aux corrélations qu'ils ont décelées, que dans le modèle qui peut les expliquer. L'espace étant un système de relations, une approche topologique ne peut avoir recours à des dichotomies qui ne sauraient être définies dans des termes relationnels. Ceci vaut particulièrement pour la différence des sexes, que les analyses de Lévi-Strauss à Désveaux ont systématiquement traitée comme une variante de la dichotomie entre culture et nature. Nous proposons ici de la concevoir comme une différence entre deux modes d'entrée en relation avec l'Autre, ce qui implique une configuration relationnelle au moins triangulaire. Pour comprendre l'étroite connexion entre la polarité des sexes et celle des saisons en Amérique du Nord, il faut considérer qu'elles correspondent toutes les deux à une polarité entre deux perspectives sur l'espace relationnel. Cette variation des perspectives, qui se matérialise de façon particulièrement claire à travers les rituels saisonniers, est l'objet de la présente étude.

\section{Le modèle topologique}

Le modèle topologique des sociétés nord-amérindiennes reposait sur les mêmes principes qu'ailleurs en Amérique (voir Hamberger 2012), à savoir une polarité sexuée associant l'intérieur à la périphérie et l'extérieur au centre. Toutefois, la variation saisonnière donnait à ces polarités une forme foncièrement dynamique : celle entre intérieur et extérieur prenait alors la forme d'une opposition entre introversion et extroversion, celle entre centre et périphérie devenait une alternance entre concentration et dispersion.

L'association de l'intérieur aux femmes et de l'extérieur aux hommes se retrouve en Amérique du Nord. Des Prairies jusqu'en Alaska, l'espace domestique était associé aux femmes. Le tipi des Plaines, la maison de terre des Prairies, la maison de nattes du Plateau, étaient construits par les femmes et leur appartenaient; on les représentait régulièrement comme corps féminin (Hassrick 1964, p. 186) ou ventre maternel (Weltfish 1965, p. 64). Cette étroite association à l'intérieur ne coupait toutefois pas les femmes de la relation à l'Autre, qu'elles étaient au contraire régulièrement appelées à médiatiser. Cependant, comme l'a premièrement constaté Lévi-Strauss (1973 [1971], p. 284-285) au sujet des sociétés du Haut Missouri, cette médiation prenait plutôt la forme « introvertie » (accueillir l'Autre à l'intérieur), tandis que sa forme « extrovertie » (se rendre chez l'Autre à l'extérieur) était plutôt confiée aux hommes.

La même logique s'applique à la dichotomie sexuée entre centre et périphérie. Loin d'impliquer une marginalisation générale des femmes, celle-ci se manifestait surtout par le fait que la dispersion donnait plus de poids à la sphère 
féminine qu'était la maison résidentielle, tandis que la concentration renforçait les relations masculines, notamment par la transmission de privilèges rituels. $\mathrm{Ce}$ modèle d'alternance entre dispersion et concentration, premièrement formulé par Mauss pour l'Arctique (1905), a été généralisé par Désveaux (1997, p. 116118 ; 2001, p. 307 et suiv., p. 321 et suiv., p. 409 et suiv.), qui, en incluant la Subarctique et les Plaines, a souligné le caractère sexué de ce qu'il décrit comme une variation entre une organisation dispersée en groupes résidentiels et une organisation concentrée de type confrérique. En effet, cette variation se matérialisait avant tout dans la topologie de l'espace sexué: si, pendant leur dispersion sur les terrains de chasse ou de pêche, les groupes résidentiels ( « bandes » des Plaines ou " maisons » de la Côte Nord-Ouest) constituaient autant de centres indépendants, ils occupaient, pendant leur concentration dans le campement tribal ou le village, des positions périphériques autour d'un centre commun (particulièrement visible chez les Algonkins des Plaines ou les Dakota, dont les «bandes » formaient des segments cohésifs dans le cercle tribal). C'est dans ce centre commun qu'émergeaient alors les édifices propres aux confréries, étroitement associées à leurs «lodges 》 ou « maisons » (Lowie 1916; Drucker 1940, p. 201). Celles-ci ne se constituaient qu'au moment de la concentration, lorsque les « sociétés d'âge » des Plaines érigeaient (en été) leurs maisons dans le cercle tribal, et que les " sociétés secrètes » de la Côte Nord-Ouest se regroupaient (en hiver) au sein de la maison cérémonielle. Or, contrairement aux maisons résidentielles, ces maisons « centrales » étaient largement un espace masculin. Même sans citer l'exemple extrême du kashim alaskien qui était une véritable maison des hommes, les protagonistes des grands rituels étaient souvent exclusivement ou majoritairement des hommes ${ }^{3}$.

Les deux polarités dynamiques (introversion/extroversion et concentration/ dispersion) pouvaient se combiner de façon différente, selon que la concentration avait lieu en été ${ }^{4}$ - comme dans les Plaines et dans la Subarctique - ou en hiver - comme sur le Plateau, sur la Côte Nord-Ouest et dans l'Arctique (voir Figure 1, page suivante).

3. Les offices dans le rituel d'hiver kwagul (Boas 1966, p. 52) et haisla (Olson 1940, p. 176) ou les danses d'esprits tlingit (Olson 1967, p. 100) étaient réservés aux hommes. Les femmes nuu-chah-nulth, bien qu'admises comme novices et danseuses lors des danses du loup, ne pouvaient pas porter les masques principaux (Ernst 1952, p. 26). Dans les Plaines, les femmes pouvaient être sponsors de la danse du soleil mais ne dansaient pas (Grinnell 1923, vol. 2, p. 220), ou seulement en remplaçant un homme (Walker 1991, p. 181). La femme sacrée blackfoot, actrice principale de la première moitié du rite, s'éclipsait pendant la «danse » proprement dite (Wissler 1918, p. 260). Même sur le Plateau, où hommes et femmes dansaient ensemble, les femmes restaient minoritaires (Ray 1933, p. 194).

4. Notons que la concentration ne concernait jamais qu'une partie de la belle saison, qui, pour le reste, était consacrée à la chasse nomade en petits groupes, donc à une « dispersion extrovertie ». 


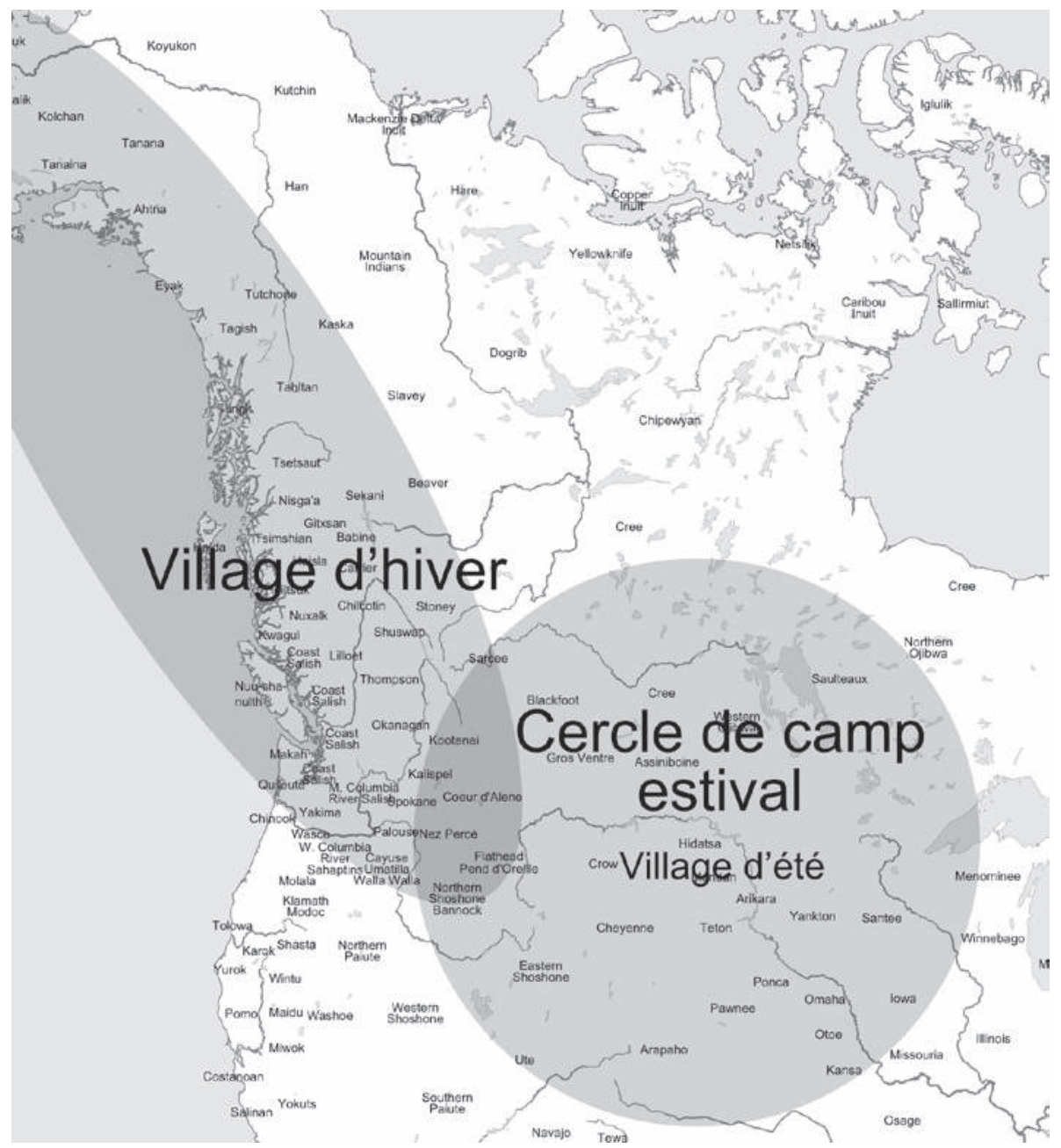

Fig. 1 - Carte de la région étudiée (fond de carte: fr.classicapaedia.wikia.com).

La concentration estivale, liée à la chasse collective au bison, coïncidait en général avec une phase de mobilité et se réalisait sous la forme d'un campement circulaire (à l'exception de la vallée du Missouri où elle donnait lieu à un village d'été). En revanche, la concentration hivernale était généralement une concentration sédentaire dans le village d'hiver, qui, lorsqu'elle ne s'opposait pas à la chasse en tant que telle (comme chez les Kwagul), était marquée par des techniques de chasse immobiles : au lieu de quitter le village pour chercher le gibier à l'instar de la chasse d'été, on attendait (dans les Plaines) que les bisons viennent au village ou (en Arctique) que les phoques montent vers le 
trou de respiration. Comme l'a souligné Lévi-Strauss (1973 [1971]), ces deux schémas de chasse correspondent à deux conceptions distinctes de la relation à l'Autre: en été, on se rendait chez l'Autre, en hiver, on le faisait venir.

De par leur caractère respectivement «extroverti » ou « introverti », l'alternance des saisons revêtait donc en elle-même un caractère sexué, qui se manifestait dans les effets sensiblement différents de l'alternance entre concentration et dispersion sur l'organisation sociale dans les Plaines et sur la Côte Nord-Ouest (voir Figure 2).

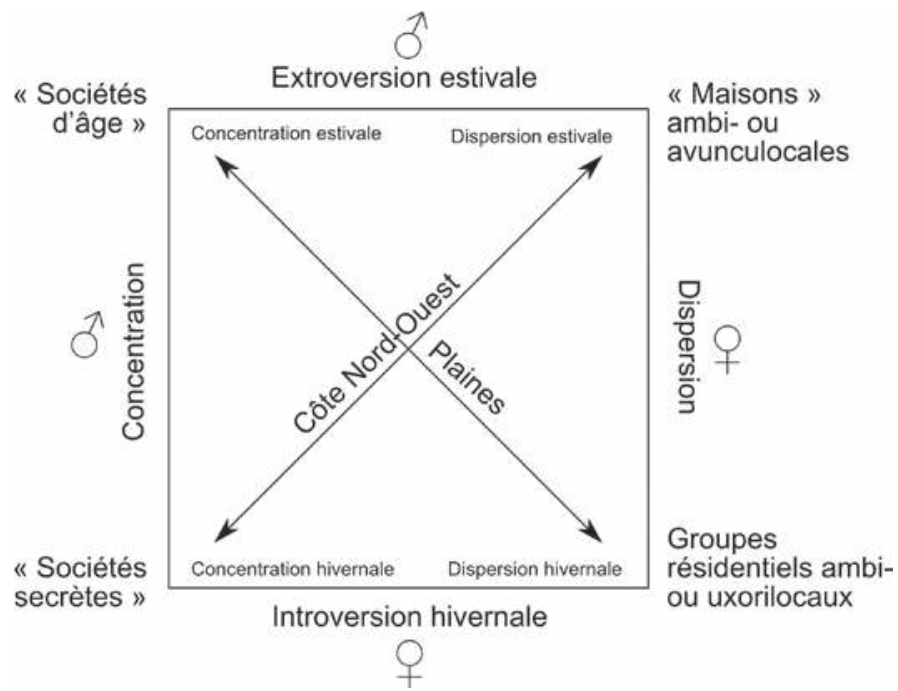

Fig. 2 - Schéma de la variation saisonnière.

Dans les Plaines, où la concentration tombait dans la saison « extrovertie » de l'été, les saisons manifestaient des valences sexuelles cohérentes selon les deux axes d'analyse. Les groupes résidentiels, généralement très souples, témoignaient pour la plupart d'une inflexion uxorilocale qui augmentait avec leur degré de sédentarité (avec les Pawnee ou les Mandan comme cas extrêmes) - le réseau social de l'hiver était tissé par des liens féminins. En revanche, les confréries estivales étaient majoritairement des sociétés d'hommes, souvent caractérisées par un principe de transmission en ligne masculine: l'affiliation aux 《 sociétés d'âge » devait être « achetée » à d'anciens membres appelés « pères » ou « grands-pères » (Lowie 1916, p. 932 et suiv.). Ces confréries pouvaient parfois se transformer en groupes de parenté patrilinéaires (voir Moore 1974 pour l'exemple cheyenne). Chez les Omaha, les groupes utérins des multiples villages d'hiver alternaient avec les clans patrilinéaires du grand campement 
tribal d'été (Fortune 1932, p. 24) $)^{5}$. De façon synthétique, la morphologie sociale des Plaines peut être caractérisée, comme l'a proposé Désveaux (2007 [2000], p. 249 et suiv.), par l'alternance entre un principe « féminin » de dispersion hivernale et un principe « masculin » de concentration estivale.

Sur la Côte Nord-Ouest, où la concentration avait lieu dans la saison « introvertie » de l'hiver, les valences sexuelles des saisons étaient plus ambiguës. Tandis que la phase de dispersion estivale était dominée par des « maisons » (au sens lévi-straussien) virilocales qui détenaient les terrains de chasse, de cueillette et de pêche, l'organisation sociale au cours de la concentration dans les villages d'hiver reposait sur des sociétés d'hommes dont l'affiliation se transmettait pourtant par les femmes. Bien que la saison de concentration constituât donc, comme dans les Plaines, le temps où s'affirmaient les liens masculins, ceux-ci, sur la Côte Nord-Ouest, impliquaient foncièrement la médiation féminine. Cette combinaison de logiques masculines et féminines pouvait prendre des formes différentes: chez les Tlingit et Haida, elles étaient conciliées en intégrant maisons et sociétés dans des clans matrilinéaires et avunculocaux. Chez les Kwagul, la hiérarchie des rangs assurait l'isomorphie entre le système de " maisons " dont les filles héritières, traitées comme des garçons, maintenaient le caractère pseudo-agnatique, et le système de « sociétés secrètes » qui se transmettaient en ligne utérine implicite, de beau-père en gendre (Boas 1920, p. 120 et suiv.; Mauzé 1989). Intermédiaires entre les deux variantes, les Heiltsuk combinaient des « maisons » ambilinéaires avec une transmission matrilinéaire des danses d'hiver (Olson 1955, p. 326-329 et 337).

Ces différences entre les variations saisonnières de la morphologie sociale des Plaines et de la Côte Nord-Ouest renvoient à une logique commune selon laquelle la différence des sexes comme celle des saisons est au fond une différence entre les formes que prend la relation à l'Autre. Ceci devenait particulièrement manifeste au comble de la concentration spatiale, lorsque les grands rites saisonniers érigeaient la relation à l'Autre (non-humain) littéralement en axe central de l'espace social. Qu'il s'agisse de la « danse du soleil » des Plaines, rite généralement printanier ou estival, ou des danses d'esprits hivernales du Plateau et de la Côte Nord-Ouest, la saison de la concentration était le moment où le modèle topologique de la société se matérialisait concrètement sous la forme d'une grande maison. Sur la Côte Nord-Ouest, la maison cérémonielle émergeait alors de la transformation d'une maison résidentielle "vidée » (Boas 1897, p. 435). La danse du soleil des Plaines, dont le nom faisait souvent référence à une « maison» ("lodge »), impliquait l'érection d'une tonnelle

5. Voir toutefois Barnes 1984, p. 178 et suiv. pour une discussion critique des règles de résidence omaha. 
cérémonielle, conçue comme un tipi géant ${ }^{6}$. Produits de la concentration, ces structures rituelles étaient systématiquement organisées autour d'un axe vertical au centre de l'espace, matérialisation de la relation à l'Autre.

Or, si, dans les deux régions, cette relation centrale était considérée comme une relation entre hommes, sa variante hivernale, caractéristique de la Côte Nord-Ouest, impliquait la médiation féminine, corrélat de son orientation « introvertie »; en revanche, la variante estivale des Plaines, qui accentuait le mode « extroverti », faisait valoir la relation masculine non médiatisée. Cela dit, nous verrons que même dans les Plaines, l'alternance entre les deux modes se retrouvait sous une forme atténuée comme variation entre rituels diurnes et nocturnes, les seconds assignant un rôle central aux femmes. En effet, l'alternance des saisons n'était que l'une des échelles où s'opérait cette variation entre modes relationnels, de sorte que le champ transformationnel de l'espace social entre les Plaines et la Côte Nord-Ouest se présente, malgré la polarisation dichotomique des saisons, comme un continuum.

\section{Le schéma spatial}

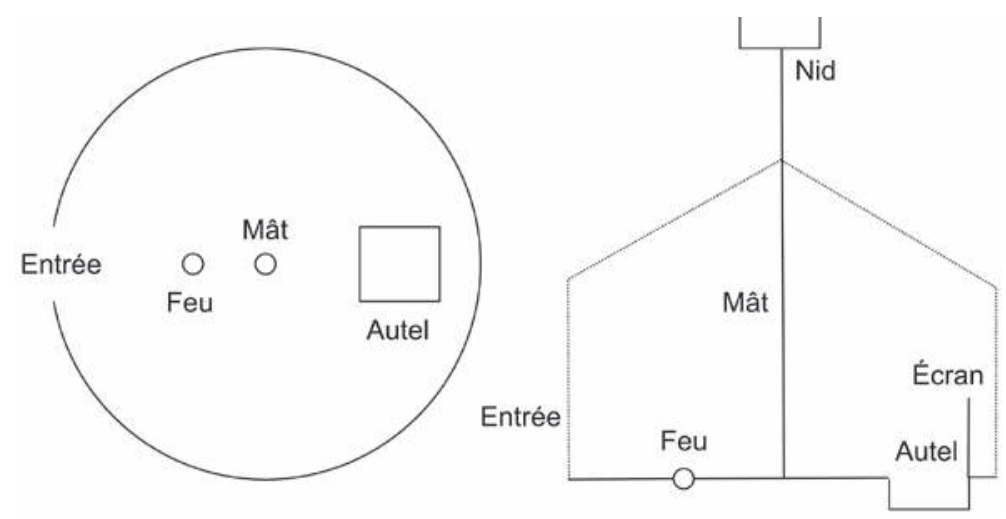

Fig. 3a-Plan schématique de la tonnelle de la danse du soleil cheyenne.

Avant de considérer cet espace sous ses différentes configurations saisonnières, considérons d'abord les principes généraux qui régissaient son architecture. La plupart des constructions (profanes et rituelles) de la région étudiée reposaient sur un dualisme concentrique entre une aire centrale commune et un pourtour divisé en secteurs familiaux ou individuels. Ce schéma se réalisait aussi bien sur un plan circulaire (tipi, maison de terre) que rectangulaire (dans les maisons

6. Les chevrons de la tonnelle de danse cheyenne portaient le même nom que les mâts d'un tipi (Dorsey 1905b, p. 114). Chez les Crow l'édifice cérémoniel partageait effectivement l'architecture d'un tipi. 

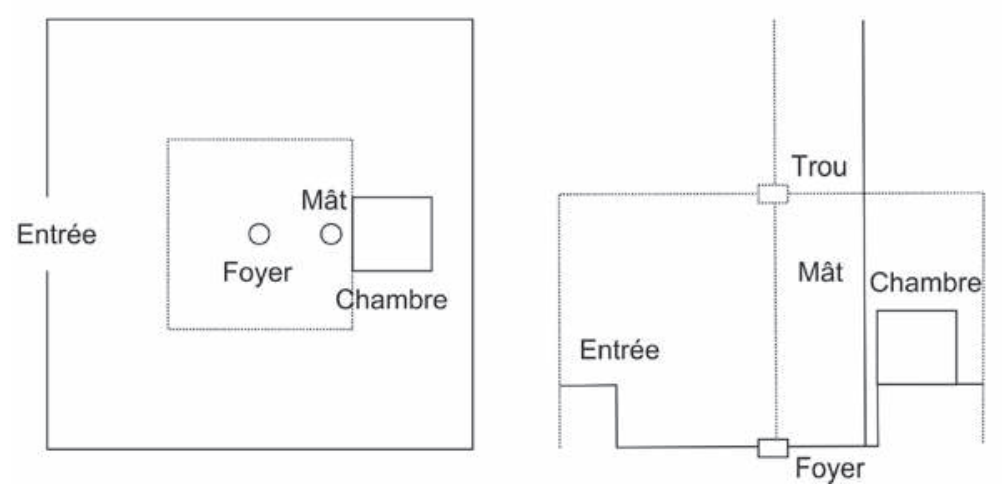

Fig. $3 b$ - Plan schématique de la maison cérémonielle kwagul.

en bois de la Côte Nord-Ouest) ${ }^{7}$. En général, le centre était le lieu du feu, ce qui vaut également pour les constructions purement rituelles comme la tonnelle de la danse du soleil. Même dans les cas où les maisons profanes n'avaient pas de foyer central (comme chez les Kwagul), celui-ci surgissait dans les maisons cérémonielles (Boas 1897, p. 370). Si le feu central servait ainsi d'indicateur de la concentration, la dispersion s'exprimait, au contraire, par la segmentation de la périphérie: les secteurs familiaux constituaient chez les Kwagul des petites maisons en miniature. Le dualisme entre centre et périphérie était donc relatif et dynamique: la concentration faisait émerger des structures centrales, alors que la dispersion divisait la périphérie en centres autonomes.

$\mathrm{Au}$ dualisme entre centre et périphérie se combinait celui entre intérieur et extérieur, qui se déclinait sur deux axes, définis par les deux ouvertures de la maison. L'un, horizontal, se dirigeait vers la porte, l'autre, vertical, vers le trou apical qui servait de cheminée (ou, en Arctique, de lucarne). Dans les Plaines, le schéma spatial était souvent orienté vers l'Est (ou vers le Sud), chez les Kwagul, il était conceptuellement orienté vers l'aval, identifié au Nord (Boas 1909, p. 415 ; 1934, p. 9). Dans les deux cas, cette orientation correspondait au lieu de l'Autre auquel s'adressaient les grands rites saisonniers - le soleil dans les Plaines, le grand cannibale chez les Kwagul (Boas 1897, p. 394) - et, dans les deux cas, cet Autre était également localisé dans les hauteurs vers lesquelles s'orientait le trou apical - qu'il s'agisse du trou du toit par lequel le danseur incarnant le cannibale kwagul pénétrait dans la maison (Boas 1897, p. 524), ou de l'ouverture géante de la loge de danse, qui permettait aux danseurs dakota de regarder le soleil (Dorsey J. 1894, p. 458). Verticalement comme

7. Même la maison longue qui servait d'édifice cérémoniel sur le Plateau peut être considérée comme un cas limite du plan concentrique, l'aire centrale étant étirée en corridor. En effet, lors des cérémonies une seule des deux portes était utilisée (Ray 1933, p. 190). 
horizontalement, le schéma spatial était orienté vers l'Autre. Il constituait de fait un modèle pour concevoir et façonner la relation à l'Autre.

Cette conception des axes horizontaux et verticaux donne une valeur particulière aux antipodes intérieurs des deux ouvertures: le foyer en bas et la place d'honneur au fond. Ceux-ci étaient souvent à leur tour associés à une sorte d'ouverture. Dans la tonnelle de la danse du soleil cheyenne ou arapaho ${ }^{8}$, le lieu situé entre le centre et le fond était souvent excavé et constituait un " autel», marqué d'un crâne de bison. C'est dans ce trou, considéré comme un tipi (Dorsey 1903, p. 120), que dansait le danseur principal. Ainsi conçu comme une entrée vers l'intérieur (et vers le bas), il n'en constituait pas moins une sortie vers l'extérieur, considéré du point de vue de l'Autre situé en haut: toujours creusé par les femmes, il représentait en effet, chez les Cheyenne et Arapaho, le trou dans le ciel que l'épouse de l'astre, selon un mythe répandu dans toutes les Plaines ${ }^{9}$, creusa afin de revenir sur terre (ibid., p. 27). Or, ce même trou dans le ciel était figuré au sommet du mât central de la danse du soleil, où l'on attachait, à côté d'un crâne de bison (ou d'une autre effigie de bison) un bâton fouisseur représentant l'instrument de son ouverture (ibid., p. 114). Il ne s'agit pas ici d'un simple effet de symétrie. L'homologie entre les ouvertures d'en haut et d'en bas apparait plutôt comme une condensation de deux systèmes de référence homologues mais décalés, permettant ainsi aux participants de la danse du soleil de se situer, selon le repère choisi, tantôt au niveau céleste (avec le trou en bas), tantôt au niveau terrestre (avec le trou en haut). Cette inscription d'une transposition virtuelle dans la structure de l'espace rituel se retrouve aussi dans la maison cérémonielle de la Côte Nord-Ouest: l'entrée du cannibale par le trou de la cheminée correspondait à sa chute mythique dans le trou du foyer, qui se trouvait effectivement au point le plus bas de l'excavation centrale (Reid 1981, p. 230) (voir Figure 4, page suivante).

Une transposition analogue s'opérait sur l'axe horizontal. Chez les Kwagul, le fond de la maison était barré par une cloison qui constituait le mur frontal de la chambre sacrée, et dont la porte figurait la gueule du monstre (Boas 1897, p. 446 $)^{10}$. Or, la chambre sacrée reproduisait la maison du grand cannibale (Reid 1979, p. 269). L'ouverture intérieure équivalait à celle vers l'extérieur. Dans la tonnelle de danse des Plaines, l'équivalent de la partition kwagul était un écran qui barrait souvent l'emplacement des danseurs (Spier 1921, p. 472).

8. Mais aussi, par exemple, dans la maison de terre pawnee, qui fonctionnait à la fois comme espace résidentiel et cérémoniel.

9. Voir Lévi-Strauss 1968, p. 161-266. Selon les versions, l'astre épousé peut être le soleil (comme dans le cas cité par Dorsey) ou la lune.

10. Dans la maison tlingit, un écran similaire constituait le mur frontal de la chambre du chef, dont celui-ci émergeait lors des rituels (Emmons 1991, p. 62; Oberg 1973, p. 53). Les novices nuu-chah-nulth étaient cachés derrière un rideau de nattes au fond de la maison (Drucker 1951, p. 395). 


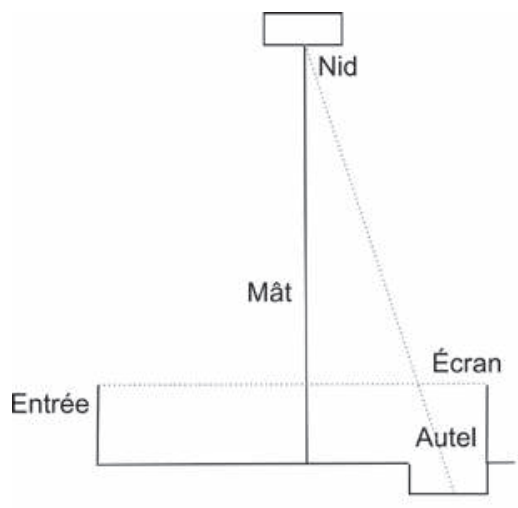

Plaines

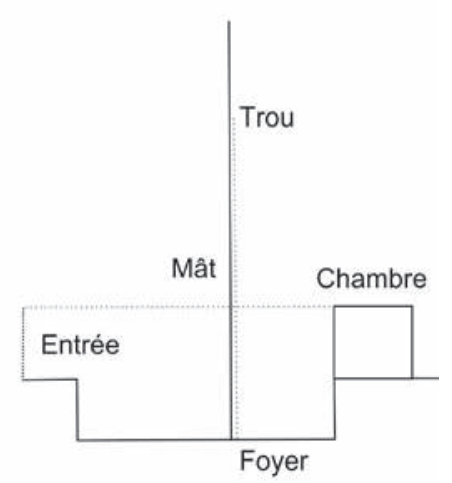

Côte Nord-Ouest

Fig. 4 - Transpositions spatiales.

Toutefois, sa valeur n'était pas la même que sur la Côte Nord-Ouest. Dans les Plaines, elle correspondait au trou d'en bas, au «tipi » dans lequel entrait le danseur principal. Sur la Côte Nord-Ouest, au contraire, elle correspondait au trou d'en haut, par lequel entrait le cannibale; et contrairement au « tipi » des Plaines qui était une excavation, la chambre sacrée de la Côte Nord-Ouest se trouvait à un niveau élevé de la maison.

L'analyse des rituels montrera que ces associations divergentes du fond de la maison - avec le bas dans les Plaines, avec le haut sur la Côte Nord-Ouest correspondent à une différence entre les points de vue adoptés par le danseur principal - celui des humains en bas ou celui des non-humains en haut. Il faut toutefois souligner que ces points de vue étaient censés changer au cours du rituel. Le rite servait précisément à effectuer ce passage d'une perspective à l'autre - passage facilité par une architecture qui établissait, à travers une série de correspondances entre ses éléments, un système de transpositions virtuelles permettant de se considérer, selon le cas, en haut ou en bas, à l'intérieur ou à l'extérieur, chez soi ou chez l'Autre.

Dans ce système de transformations, une valeur toute particulière échoit à l'axe vertical, matérialisé par le seul élément architectural qui se trouvait exclusivement dans les constructions rituelles : le mât central. Le mât central de cottonwood ou de saule était un élément quasiment universel de la danse du soleil des Plaines et de ses homologues ${ }^{11}$. Sur le Plateau, on installait un conifère au

11. Chez les Crow il correspondait au premier mât du tipi (Lowie 1915, p. 29). Les Mandan plaçaient le mât représentant l'ennemi au sommet de la maison cérémonielle (Maximilian 1843, p. 342-344, cité in Bushnell 1922, p. 132). Même chez les Pawnee, où tous les rituels majeurs impliquaient quatre mâts, le concept d'un mât central liant le trou apical et le foyer restait palpable: la première maison de terre était, selon le mythe, 
centre de la maison cérémonielle (Ray 1933, p. 190 et suiv.; Turney-High 1933, p. 106). Les Kwagul et leurs voisins septentrionaux érigeaient un mât de cèdre au fond de la maison du rituel d'hiver, qui dépassait du toit (Boas 1921, p. 1244).

Quel est le sens de cet élément distinctif de l'architecture rituelle? Certes, d'abord, celle d'établir la communication entre le haut et le bas. Les Arapaho l'identifiaient à l'arbre par lequel l'épouse de l'astre ${ }^{12}$ était montée au ciel (Dorsey 1903, p. 112), les Kwagul au poteau de cuivre par lequel le cannibale descendait sur la terre (Boas et Hunt 1905, p. 401 ; Müller 1955, p. 18 et suiv.). Mais sa signification est bien plus précise. Dans les Plaines comme sur la Côte Nord-Ouest, le mât central, élément « étranger » aux maisons profanes et qui devait y être intégré par l'extérieur, représentait l'ennemi. Presque partout dans les Plaines il était traité comme un ennemi (Spier 1921, p. 465), parfois même nommé « ennemi » (Dorsey 1905b, p. 116). Les Kwagul l'appelaient « poteau de cannibale » et attachaient à son sommet une effigie du cannibale (Boas 1897, p. 446 ; 1930, p. 107 et suiv.), comme le faisaient les Dakota (Walker 1917, p. 108 et suiv.). Dévorateur d'humains, cet ennemi était toujours un être céleste, souvent identifié aux astres ou aux oiseaux prédateurs. Les Cheyenne identifiaient le mât au soleil (Dorsey 1905b, p. 112), les Arapaho, qui considéraient la danse du soleil comme une « partie jouée contre le soleil » (Dorsey 1903, p. 151), y voyaient «l'homme d'en haut » (ibid., p. 112). En même temps, le mât était le perchoir d'un oiseau (aigle ou oiseau tonnerre) dont un fagot de broussailles attaché au sommet représentait le « nid ». Sur le Plateau, il servait de perchoir à la chouette, esprit-gardien du chamane (Ray 1933, p. 191). Issu des montagnes (Boas 1897, p. 394), le grand cannibale qui faisait irruption dans le rituel d'hiver de la Côte Nord-Ouest, dont un des noms était « apporté d'en haut » (ibid., p. 418), était représenté comme un oiseau prédateur (Reid 1979, p. 257).

L'axe vertical du rituel liait donc invariablement les humains en bas avec un ennemi non-humain en haut. Toutefois, les danseurs sur lesquels le rituel était censé opérer pouvaient adopter les deux perspectives, et c'est précisément ce changement de perspective que le rituel effectuait, quoique pas toujours dans la même direction. Dans la danse du soleil des Plaines, il s'agissait de transmettre aux candidats la puissance de l'ennemi surnaturel. Dans le rituel d'hiver de la Côte Nord-Ouest, il s'agissait au contraire de ramener le novice qui incarnait l'ennemi surnaturel à l'état d'être humain. La transformation de perspective opérait donc de bas en haut dans le rite estival, de haut en bas dans le rite hivernal, correspondant aux orientations de la chasse qui consistait à se rendre chez l'Autre en été et à le faire venir en hiver. Dans les sections suivantes, nous considérerons de plus près comment cette transformation opérait dans les deux cas.

construite autour d'un frêne central que le soleil brûlait, laissant le trou du foyer dans le sol (Chamberlain 1982, p. 159).

12. En l'occurrence de la lune. Voir supra, note 9. 


\section{Le modèle estival : de bas en haut}

\section{Du point de vue de la proie}

La relation antagoniste centrale autour de laquelle tournait la danse du soleil des Plaines était conçue sur le modèle des deux principales activités estivales - guerre et chasse au bison. L'abattage de l'arbre destiné à servir de mât central était mis en scène comme une expédition de guerre. Toutefois, dès cette campagne qui précédait et accompagnait la construction de la tonnelle de danse, les perspectives semblaient s'inverser: les éclaireurs qui revenaient étaient accueillis comme des ennemis (Dorsey 1903, p. 78; Lowie 1915, p. 28), les femmes qui montaient les chevaux de leurs cavaliers étaient traitées comme des captives (Mandelbaum 1940, p. 267), les simulacres d'attaque visaient parfois le propre camp des assaillants (Walker 1991, p. 178; Dorsey 1905a, p. 73). Une fois la tonnelle construite, l'inversion était complète : l'arbre capturé par les humains apparaissait désormais comme l'instrument de leur captivité, voire comme leur capteur. Ayant apporté l'ennemi à la maison, les humains se retrouvaient dans la maison de l'ennemi.

Ce fait se manifestait de façon exemplaire dans les mortifications que certaines variantes de la danse du soleil imposaient aux danseurs, et que l'ethnographie des Plaines synthétise sous le nom de « torture ». Systématiquement précédée par la récitation ou la mise en scène des exploits de guerre, cette « torture » consentie reproduisait effectivement le traitement des captifs, tout en se rapprochant du cannibalisme : chez les Hidatsa, dont le rituel s'adressait aux cannibales célestes du «peuple d'en haut », elle représentait la torture du héros mythique lors de sa captivité céleste (Bowers 1965, p. 306 et suiv.). La forme principale de torture consistait à attacher (parfois à suspendre) les candidats au mât central avec des lanières qui perçaient la chair de la poitrine ou du dos. Outre l'idée de la captivité, cet attachement matérialisait plus largement le lien avec l'ennemi, représenté par l'effigie ou le crâne de bison placé au sommet du mât. Le bison, gibier principal, était ici en position de prédateur, fortement associé au soleil ${ }^{13}$, tandis que les humains se trouvaient dans la position de gibier. La maison cérémonielle des Mandan représentait le lieu où l'aigle moucheté avait enfermé les animaux (Bowers 1950, p. 113).

En effet, comme l'a noté Spier (1921, p. 497, note 3), l'architecture de la loge de danse correspondait étroitement à celle d'un enclos de bisons ("pound»), qui, dans sa version cree par exemple, comprenait aussi un arbre central et un crâne de bison; de jeunes hommes munis de sifflets d'os d'aigle rabattaient le

13. Pour les Arapaho, « Young Bull » (incarné par le crâne) était le petit enfant du soleil (Dorsey 1903, p. 115), pour les Dakota le soleil résidait la nuit chez le bison, sous la terre (Walker 1917, p. 83). 
troupeau vers l'entrée (Mandelbaum 1940, p. 190 et suiv., voir Figure 5). Les rituels de battue (" pounding ») faisaient partie de la danse du soleil kiowa et comanche. Certes, le pounding, qui consistait à faire venir les bisons, était une technique de chasse hivernale, alors que la danse du soleil se déroulait avant l'expédition estivale chez les bisons. Mais du point de vue des bisons considérés comme prédateurs, cette expédition apparaît bien comme un pounding des humains.

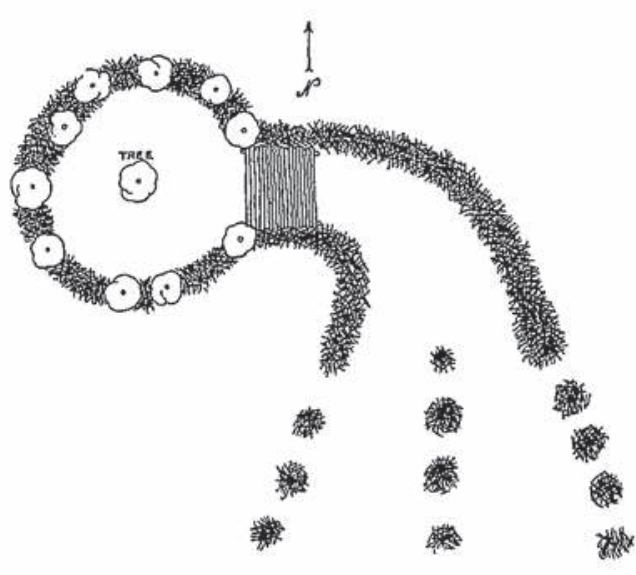

Fig. 5 - « Buffalo pound »

(Mandelbaum 1940, p. 190).

L'inversion des rôles de prédateur et de proie se dessinait depuis le début: si les candidats étaient symboliquement attachés au crâne de bison, on attachait aussi souvent des crânes de bisons aux candidats, et presque partout ceux-ci étaient munis de sifflets d'os d'aigle, généralement utilisés par des guerriers attaquants (Will et Spinden 1906, p. 123), et dont le bruit représentait le cri de l'oiseau prédateur (Black Elk 1953, p. 71; Dorsey 1905b, p. 154). Toutefois, cette inversion restait en quelque sorte en latence: comme le disaient les Comanche, les danseurs ne représentaient pas tant des aigles que des aiglons encore incapables de voler (Linton 1935, p. 426). Bien qu'orientés vers le haut, ils restaient assignés en bas. Les imitateurs d'oiseaux qui, chez les Crow, montaient au mât, n'étaient jamais des candidats à la torture, mais des hommes que leur position rituelle de transféreur ou leur lien individuel avec l'aigle ou le soleil assimilait à des êtres célestes. Cependant, la voix du sifflet, comme la lanière perçant la chair $^{14}$, représentaient moins une position qu'une relation, dont l'inversion constituait toujours une possibilité latente et le but ultime du rituel.

14. Sur l'association entre lanière et sifflet voir aussi Désveaux 2001, p. 106, 110-111. 
Le caractère cannibale de cette relation était mis en évidence par une autre forme de torture, qui consistait à se (faire) couper des morceaux de chair ou des doigts. Chez les Hidatsa, les doigts coupés servaient généralement d'offrandes au soleil cannibale (Bowers 1965, p. 322). Mais le cannibalisme du soleil s'exprimait surtout dans l'épreuve fondamentale et presque universelle de toutes les formes de la danse du soleil : à savoir la soif, qui a donné son nom (" thirsting-dance ») au rituel chez les Ute-Shoshone et les Algonquins du Nord. Être mangé par le soleil s'exprimait avant tout par le fait de ne pas boire. C'est en extrayant l'eau de leurs corps brûlés que le soleil dévorait les candidats.

Cette relation aussi était susceptible de s'inverser progressivement. Chez les Shoshone et Cree, les danseurs utilisaient leurs sifflets pour aspirer de l'eau sur le mât central (Lowie 1919, p. 402; Mandelbaum 1940, p. 270). Ailleurs, ils soulageaient leur soif en mâchant de l'écorce de cottonwood, donc du bois dont était fait le mât. Le rituel visait le passage d'un pôle relationnel à l'autre: du gibier au prédateur, du captif au guerrier victorieux. Qui plus est, cette inversion était inhérente à la relation elle-même, qui était en effet pensée comme un transfert de puissance. C'est pour acquérir la puissance du prédateur que le candidat se mettait en position de gibier. La danse du soleil était foncièrement un rite de transmission.

\section{La transmission du regard}

Cette transmission concernait d'abord le rituel en tant que tel. Le privilège de la danse du soleil appartenait fréquemment à un groupe de tuteurs (généralement d'anciens danseurs) auxquels les candidats devaient l' " acheter » ou par lesquels ils devaient se faire « adopter ». Dans le cas où le privilège de danse était héréditaire, une relation d'adoption s'établissait entre le danseur principal et le propriétaire de la danse (Lowie 1915, p. 12), et même si la danse était librement accessible, la relation aux tuteurs restait importante (Kroeber 1908, p. 265). Comme dans le cas des sociétés d'âge, le transféreur était conçu comme « grand-père » ou « père ». Chez les Mandan et Hidatsa, il s'agissait du père réel.

En même temps, ce lien de filiation entre le candidat et son tuteur était assimilé au lien qui le reliait à l'ennemi destinataire du rite. Chez les Arapaho et Cheyenne, c'étaient les tuteurs qui perçaient la chair des candidats; chez les Dakota, ce rôle incombait aux anciens danseurs (ici distincts des tuteurs); chez les Mandan et Hidatsa, aux pères classificatoires des candidats. Inversement, les Dakota considéraient le soleil comme «père »(Walker 1917, p. 83). L'éclaireur cree ayant repéré l'arbre l'annonçait au danseur principal en lui apportant alternativement les salutations de l'ennemi ou celles de son père (Mandelbaum 1940, p. 266). Si les danseurs prenaient donc la position de victimes capturées, attachées et torturées, alors que leurs pères ou grands-pères 
spirituels adoptaient celle des prédateurs, c'était dans le but de conférer à leurs proies la position de prédateur.

Cette transformation du point de vue était effectivement opérée par une action sur les yeux. C'est en fixant des yeux un crâne de bison que les danseurs dakota se transformaient en hommes-bisons (Walker 1917, p. 115). Qui plus est, cette transformation n'était accomplie (car reconnue par les anciens hommes-bisons qui les torturaient) qu'au dénouement d'une torture qui visait elle-même les yeux. En effet, en plus du percement de la chair et de la privation d'eau, l'épreuve de la danse du soleil consistait surtout à fixer l'Autre du regard. Chez les Dakota et Ponca, cela impliquait de fixer le soleil (d'où le nom dakota du rituel, "sungazing-dance »). Chez les Arapaho, il fallait fixer un point sur le mât souvent si haut qu'on semblait regarder le soleil (Hilger 1952, p. 149); chez les Crow, une poupée qui représentait la « face du soleil » (Lowie 1915, p. 30). Opérant le lien entre prédateur et victime, le rayon du soleil qui perçait, réellement ou symboliquement, les yeux du danseur, avait la même fonction que les lanières qui perçaient sa chair, et avec lesquelles il semblait parfois se confondre - dans une variante ancienne de la torture chez les Cheyenne, on attachait des lanières juste en dessous des yeux (Dorsey 1905b, p. 176, voir Figure 6).

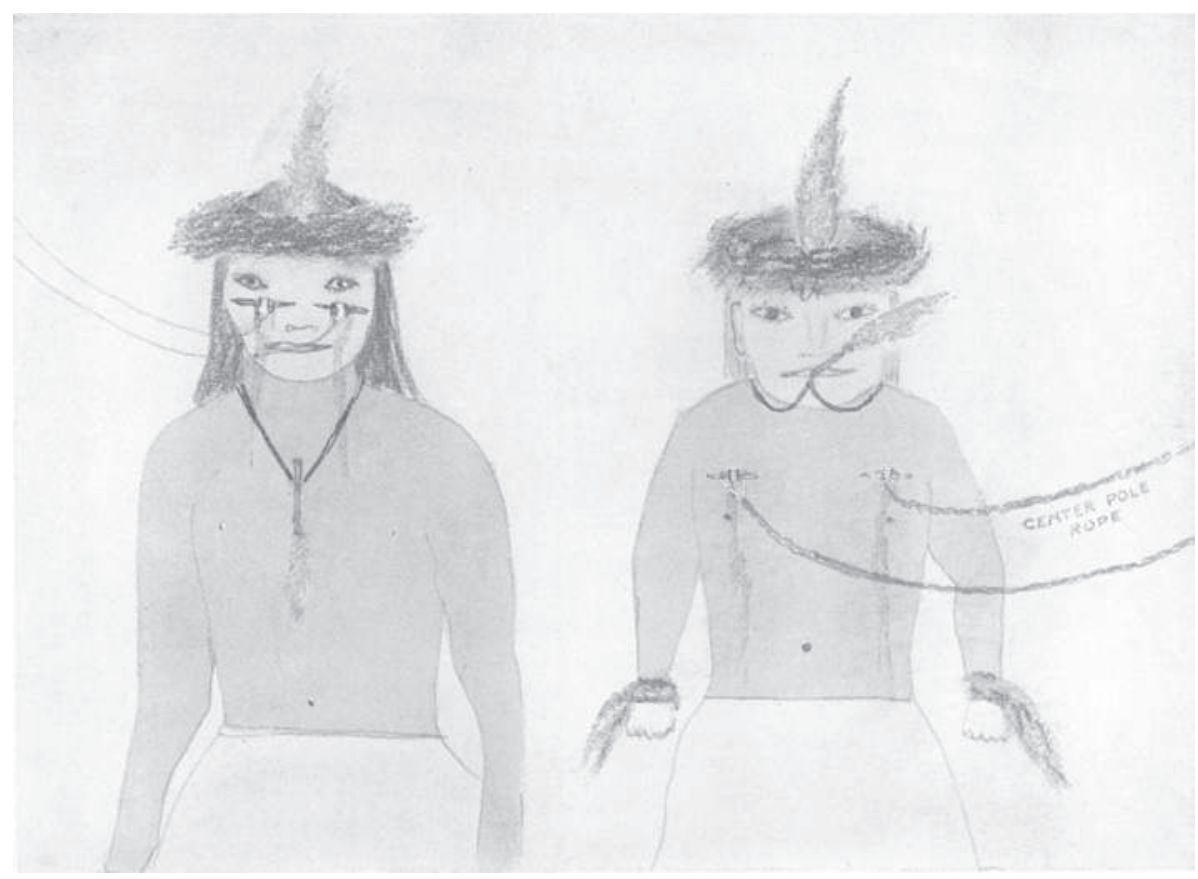

Fig. 6 - Ancienne torture cheyenne (Dorsey 1905b, pl. LXV). 
L'action du soleil sur le candidat consistant surtout à le vider de son eau, on pourrait même interpréter les larmes dessinées par les Arapaho sous les yeux des candidats (Dorsey 1903, p. 172) comme une expression synthétique de l'attaque des yeux - percés, assoiffés, aveuglés par le regard de l'ennemi.

Cet aveuglement menait à la clairvoyance. La danse du soleil, comme d'autres techniques impliquant l'auto-torture, la fixation du soleil ou la danse devant un mât (voir Spier 1921, p. 492 et suiv.; Mandelbaum 1940, p. 252), avait pour but l'obtention d'une vision, conçue (chez les Dakota) comme une communication du soleil (Walker 1917, p. 61). Or, parmi les visions attendues figurait celle d'un ennemi tué - ainsi chez les Gros Ventre et Crow, où le rituel aboutissait à une expédition de guerre réelle (Kroeber 1908, p. 265 ; Lowie 1915, p. 50) ${ }^{15}$. Ainsi s'achevait l'inversion de la perspective: le regard percé devenait regard perçant, la vision de l'ennemi torturant qu'est le soleil basculait en vision de l'ennemi torturé, et parfois même le mât devant lequel on dansait en sautillant se transformait en danseur: chez les Cree d'Alberta, il était censé sautiller au comble du rituel (Goddard 1919, p. 304).

\section{La médiation féminine}

Jusqu'à présent nous avons considéré la relation centrale de la danse du soleil comme un lien exclusivement masculin. Pourtant, le rituel attribuait un rôle important aux femmes. Tout d'abord, il incombait le plus souvent aux femmes d'abattre l'arbre qui devait servir de mât central. Chez les Mandan, qui ne construisaient pas de tonnelle de danse temporaire, elles brisaient le bâton de l'acteur représentant le cannibale solaire (Catlin 1867, p. 23 et suiv.), muni, comme les effigies d'ennemi aux sommets des mâts dakota ou cheyenne (Walker 1917, p. 108; Dorsey 1905b, p. 97), d'un pénis surdimensionné (Bowers 1950, p. 145). L'antagonisme guerrier revêtait ainsi des connotations sexuelles explicites.

Cet aspect sexuel de l'antagonisme guerrier correspondait à l'aspect guerrier de l'antagonisme sexuel. Les sociétés des Plaines qui valorisaient la chasteté féminine encourageaient en même temps les hommes à la briser. Des conquêtes érotiques valaient comme des exploits guerriers et étaient parfois comptabilisées comme tels (Will et Spinden 1906, p. 132; Hassrick 1964, p. 45, 111; DeMallie 1983, p. 252 et suiv.). Les Dakota dénonçaient les femmes infidèles publiquement lors de la danse du soleil (Deloria, ms. 1, 109 in DeMallie 1983, p. 254). Inversement, les femmes vertueuses cheyenne ou blackfoot dévoilaient pendant la danse du soleil publiquement les noms des hommes ayant tenté en vain de les séduire (Dorsey 1905b, p. 157 et suiv.; Wissler 1918, p. 240). La chasteté féminine était une vertu guerrière, proclamée avec la même posture

15. Contrairement aux Gros Ventre, le visionnaire crow n'était pas lui-même torturé ; toutefois, la torture des autres danseurs s'arrêtait au moment même où la vision survenait. 
rituelle que les exploits militaires des hommes. Or, les femmes chargées d'abattre l'arbre étaient toujours des vierges ou des épouses fidèles.

Toutefois, la vertu de l'épouse fidèle ne consistait pas seulement à refuser des rapports sexuels proposés par l'ennemi. Elle était aussi la condition pour qu'elle puisse les lui proposer, avec l'accord et au profit de son mari. Dans les Plaines, le coït rituel avec le « grand-père » ou « père » du mari était une forme répandue de transmission de puissance du premier au second. Chez les Mandan et Hidatsa, le coït rituel avec le père classificatoire du mari, strictement réservé aux femmes fidèles et non remariées, accompagnait tout transfert de puissance spirituelle, y compris celle des bisons que l'on cherchait à attirer lors du rituel du « bâton rouge », pendant hivernal de la danse du soleil (Bowers 1950, p. 317 et suiv.; 1965, p. 455 et suiv.; Lowie 1919, p. 416). Ces connotations « hivernales » du coït rituel renvoient au mode relationnel qu'il accentue : séduisant l'Autre plutôt que de le pourchasser, il s'inscrivait avec plus de facilité dans les rites hivernaux, qui servaient justement à faire venir les bisons au sein du village $^{16}$. Toutefois, on retrouve le coït rituel dans le contexte du rite estival, notamment, mais non exclusivement, chez les groupes algonquins ${ }^{17}$.

Ainsi, les Blackfoot, qui le pratiquaient pour transmettre les sociétés d'âge, voyaient une nette équivalence entre le rôle de l'épouse cédée au « père » spirituel et celui de la femme sacrée (obligatoirement chaste) qui - trait singulier de ce groupe - organisait la danse du soleil (Kehoe 1995, p. 119 et suiv.). Cette danse provenait, selon leurs mythes, d'un cerf qui l'avait reçue d'un rival comme récompense pour avoir couché avec sa femme, et le geste de la femme sacrée lors de l'abattage de l'arbre répétait celui de la femme-cerf (Ewers 1958, p. 178; Wissler 1918, p. 256).

Chez les Arapaho et Cheyenne, le coït rituel était intégré dans la danse du soleil en tant que rite nocturne - l'ennemi d'en haut apparaissait alors sous l'aspect de la lune (voir Dorsey 1903, p. 173, 1905, p. 130 et suiv.). De même que son volet diurne centré sur la « lumière du soleil du monde entier » (nom que les Cheyenne donnaient au mât central) (Dorsey 1905b, p. 111), cette partie nocturne du rituel médiatisait un transfert de puissance, en l'occurrence matérialisée par un morceau de racine que le « grand-père » crachait pendant le coït dans la bouche de la femme, et que celle-ci transférait ensuite par la bouche à son mari qui le mâchait et s'enduisait avec le crachat. La racine étant l'équivalent du sperme (Dorsey 1903, p. 173), il s'agissait d'une fécondation indirecte du « petit-fils » par le $«$ grand-père $»{ }^{18}$. Mais la pratique de s'enduire

16. Dans toutes ses variantes, le coït rituel était conçu comme un coït avec les bisons.

17. Sans l'intégrer comme méthode de transfert, l'Okipa des Mandan était également clos par un coït rituel avec les « hommes bisons ", dirigé par la femme ayant brisé le bâton du représentant terrestre du soleil (Catlin 1867, p. 33 et suiv.).

18. Sur ce thème voir déjà Lévi-Strauss 1958 [1956]. 
avec le crachat des « grands-pères » existait aussi dans la phase diurne du rituel. Chez les Cheyenne, les « grands-pères » vivifiaient les danseurs en crachant une racine mâchée sur une herbe qu'ils faisaient ensuite passer entre les lèvres de ces derniers (Dorsey 1905b, p. 151). De façon générale, la salive contenant la racine constituait une forme de bénédiction (Dorsey 1903, p. 43, 1905, p. 125). Toutefois, elle était susceptible de rendre aveugle lorsqu'elle pénétrait dans les yeux (Dorsey 1905b, p. 74). À la fois fécondant et aveuglant, le crachat du « grand-père » était analogue au rayon du soleil.

Tout comme la torture pratiquée par le représentant de l'astre, le coït rituel avec ce dernier avait pour but de lui retirer sa puissance - le mythe Hidatsa le décrit comme une façon de piéger le soleil (Bowers 1965, p. 452 et suiv.) - et était, par conséquent, plutôt évité par les hommes (Lowie 1913, p. 304). Chez les Arapaho du Nord, où le coït rituel restait symbolique, le « grand-père » se gardait même de regarder la femme qui présentait son corps à la lune, se contentant de demander au " grand-père d'en haut» de fixer son regard sur elle (Kroeber 1908, p. 244, note 1). Ainsi que la torture, le coït rituel était une affaire de regard - mais contrairement à la torture, il ne s'agissait pas de fixer du regard l'astre représentant l'ennemi en haut, mais au contraire d'attirer le regard de celui-ci sur la femme qui s'allongeait en bas.

L'analogie entre le coït rituel et la torture en tant que variantes du transfert de puissance - l'un médiatisé et nocturne, l'autre immédiate et diurne - n'est pas seulement formelle. Les deux étaient accompagnés des mêmes gestes et formules. Comme le tortureur, le partenaire du coït rituel récitait ses exploits de guerre (Bowers 1950, p. 318), et comme le candidat à la torture, la femme passait ses mains sur les bras du partenaire afin de s'approprier sa puissance (Lowie 1913, p. 229; McLean 1888, p. 235-237 cité in Wissler 1918, p. 264). Coït rituel et torture pouvaient même se confondre, comme l'illustre la variante extrême de la danse du soleil que représente le sacrifice à l'étoile du matin des Pawnee (Dorsey 1906; Murie 1989; Linton 1922). Ce rite culminait dans l'abattage d'une jeune captive attachée à un arbre par un guerrier représentant l'étoile, qui la pénétrait avec une flèche, suite à quoi tous les hommes (jusqu'aux plus petits garçons) tiraient leurs flèches dans le corps de la fille. Modèle d'une transmission spirituelle aux hommes par l'intermédiaire d'une femme, ce rite mettait en scène un coït rituel mythique (Thurman 1983, p. 160) en même temps qu'il inversait l'abattage de l'arbre par la captive au début de la danse du soleil.

Le parallélisme entre torture et coït rituel reste éclairant lorsqu'on le considère dans l'autre sens. L' " eau suave » avec laquelle les danseurs arapaho et cheyenne s'allégeaient à la fin du rituel (Dorsey 1903, p. 145; 1905, p. 155) représentait le sang menstruel, résultat d'un rapport sexuel avec la lune, et assimilé au sang d'animaux bouilli, un mets favori des Arapaho (Dorsey 1903, p. 177 et suiv.). D'un être saignant et assoiffé, le danseur était devenu un buveur de sang. Notons que les Arapaho considéraient les danseurs suspendus 
au mât comme homologues de l'épouse de la lune lors de sa descente du ciel (ibid.). La spécificité des variantes « hivernales » et « nocturnes » de la danse du soleil consistait à introduire la femme explicitement comme troisième terme pour médiatiser cette relation. C'est en attirant l'ennemi d'en haut (plutôt que d'être capturées par lui) que les femmes parvenaient à désaltérer, vivifier et féconder les hommes d'en bas (rôle qui leur incombait aussi aux marges du rituel diurne $\left.{ }^{19}\right)$.

En résumé, le triangle relationnel reliant les hommes, les femmes et l'Autre prenait dans les Plaines la forme d'un axe central, vertical et asymétrique, mais en même temps pensé comme une ascension permettant à l'homme d'en bas de remplacer son « père » ennemi d'en haut. Ce lien de substitution, vecteur de filiation spirituelle, s'établissait de façon directe et « diurne » comme un acte de dévoration (mutilation, dessèchement, aveuglement), mais pouvait aussi se construire, de façon indirecte et « nocturne », par l'intermédiaire du double lien de contiguïté qu'entretenait la femme avec les deux adversaires, avançant ainsi de la périphérie vers le centre du rituel (voir Figure 7, où les flèches indiquent l'orientation du regard).

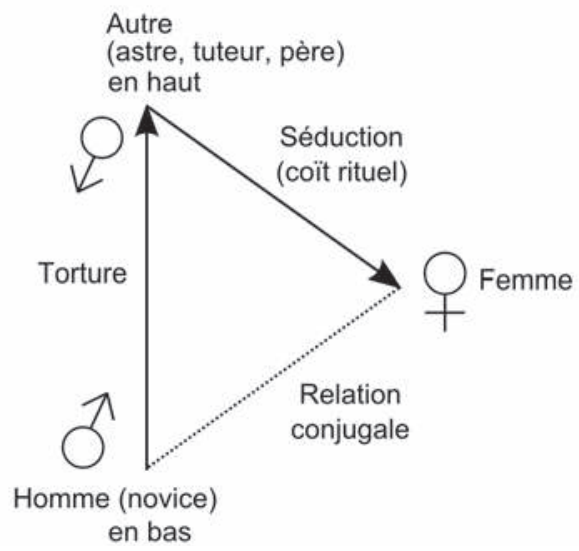

Fig. 7 - Triangle relationnel (Plaines).

Le degré d'intégration de la médiation féminine à la danse du soleil des Plaines - comme prélude, finale ou contrepoint - variait d'une société à l'autre ${ }^{20}$, mais partout elle s'inscrivait dans une inversion du rapport à l'ennemi céleste,

19. Chez les Dakota, les filles pouvaient tempérer la soif de leurs favoris en leur apportant des écorces de cottonwood mâchées (Walker 1917, p. 118; Deloria 1929, p. 400). L'écorce (de la même espèce que le mât central) sert ici de version atténuée de la racine mâchée des Algonquins.

20. Pour une étude de ces variations dans leurs rapports avec l'organisation sociale voir Désveaux 2007 [2000]. 
dont la puissance était brisée ou absorbée par les femmes. Quel rôle jouait alors la médiation féminine dans les régions où les grands rituels collectifs étaient d'emblée fondés sur la relation inverse, c'est-à-dire sur la capture et le désarmement de l'ennemi surnaturel? C'est pour répondre à cette question que nous allons à présent nous tourner vers les fêtes d'hiver du Nord-Ouest.

\section{Variations hivernales: de haut en bas}

\section{Le Plateau}

La transformation entre les modèles estival et hivernal peut être étudiée concrètement sur le Plateau, où les deux versions du rituel se côtoyaient. La danse d'esprits du Plateau, hivernale et nocturne, correspondait étroitement à la danse du soleil, bien connue de ces sociétés et pratiquée par l'une d'entre elles (les Kutenai). Les deux rituels se ressemblaient à beaucoup d'égards (voir Ray 1939, p. 123 et suiv.). Dans les deux cas, le danseur principal se trouvait au centre; la danse consistait en sautillements ininterrompus et il était interdit aux danseurs de manger et de boire (Ray 1933, p. 193 et suiv.; 1937, p. 598; Turney-High 1933, p. 103 et suiv.; 1937, p. 39). La maison de danse était, dans les deux cas, centrée sur un mât dont le sommet représentait un point de vue supérieur: si les Kutenai faisaient grimper un officiant sur le mât central de la danse du soleil pour agir comme « guetteur » (Turney-High 1941, p. 180), le chaman de la danse d'esprits sanpoil attachait un arbre au toit pour y faire siéger la chouette représentant ses propres yeux (Ray 1933, p. 191).

Une première différence par rapport aux Plaines consistait en ce que la communication avec l'être surnaturel ne se produisait pas à la fin mais au début de la danse d'esprits, et non pas sous la forme d'une vision mais d'une incarnation de l'esprit par le danseur (Ray 1939, p. 114). Les danseurs se rendaient à tour de rôle au mât central, le saisissaient et représentaient les actions de l'esprit (Ray 1933, p. 194 et suiv.). Or, lorsque l'esprit gardien était le geai bleu, ce déplacement s'effectuait non seulement de la périphérie vers le centre, mais aussi du bas vers le haut: criant comme les oiseaux qu'ils incarnaient, les « geais bleus » grimpaient sur les chevrons, avant de s'enfuir sur un arbre éloigné. Il incombait alors aux autres danseurs, restés en bas, de les capturer et de les ré-humaniser à l'aide d'encens ou par un traitement chamanique (Ray 1933, p. 191 et suiv.; 1937, p. 595-599; 1939, p. 121 et suiv.). Redevenus humains, ils recevaient de l'eau sacrée (Turney-High 1933, p. 105; 1937, p. 40). L'opération se déroulait donc à l'inverse de la danse du soleil : dans cette dernière (version kutenai), les danseurs étaient « capturés » au début, dansaient « en bas » et étaient finalement « libérés» (Turney-High 1941, p. 181 et suiv.). Dans la danse d'esprits, ils s'enfuyaient au début, montaient « en haut» et devaient être capturés à la fin. 
Malgré ces inversions, la relation entre oiseaux et humains gardait les traits qui la caractérisaient dans la danse du soleil. Certes, les geais bleus ne torturaient pas les humains, toutefois ceux qu'ils touchaient s'évanouissaient (TurneyHigh 1933, p. 105). Plutôt que prédateurs cannibales, ils étaient charognards et voleurs de nourriture ${ }^{21}$. Toutefois, leur façon de priver les autres de nourriture - et de veiller ainsi sur le respect du jeûne - frôlait le cannibalisme : ils pouvaient retirer la nourriture de la gorge d'une personne pour la manger (Ray 1933, p. 191). Dans les variantes spokane ou kalispel où les geais bleus étaient des chamanes, cette capacité de pénétrer à l'intérieur de l'autre pour en extraire des éléments assumait une fonction thérapeutique (Ray 1937, p. 597 et suiv.). Surtout, elle correspondait à une forme de clairvoyance : les éléments « extraits » incluaient les secrets autant que les objets matériels. Les geais bleus pouvaient lire les pensées, voir des personnes éloignées, retrouver des objets perdus et prédire l'avenir (Turney-High 1933, p. 105; 1937, p. 40; Ray 1933, p. $191 ; 1937$, p. 597, 599).

La transformation entre les rituels estival et hivernal du Plateau préservait donc aussi bien la relation entre non-humains en haut et humains en bas, que celle entre initiateurs capteurs et initiés capturés, mais elle inversait leurs positions respectives : dans le rituel d'hiver, c'étaient les êtres d'en bas qui capturaient ceux d'en haut. Par rapport à la danse du soleil des Plaines, l'inversion n'était pas complète: aucune transmission n'avait lieu entre capteurs et capturés, l'esprit du geai bleu étant parfaitement individuel. Mais lorsqu'on passe à la Côte Nord-Ouest, non seulement l'inversion s'achève, mais la relation centrale regagne toute la force qu'elle possédait dans les Plaines sous des signes inverses : elle se présente alors comme un lien entre des capteurs dévorés en bas et des novices cannibales en haut.

\section{La Côte Nord-Ouest}

Sans doute d'origine heiltsuk, les danses des esprits cannibales étaient pratiquées par les Kwagul (Boas 1897, p. 500 et suiv.), les Wuikinuxv, Heiltsuk et Haisla (Drucker 1940), les Haida (Swanton 1905, p. 155 et suiv.) et les Tlingit méridionaux (Swanton 1908, p. 436). Chez les Haida, elles s'intégraient dans les potlatchs funéraires. Sous une forme particulière (qui transforme les cannibales en loups), elles se retrouvent également chez les Nuu-chah-nulth (Ernst 1952; Boas 1891, p. 599 et suiv.).

Chez les Kwagul (sur lesquels nous nous concentrerons par la suite), elles constituaient la dernière phase du rituel d'hiver, processus initiatique qui commençait avec l'enlèvement du novice par les esprits cannibales - reconnaissables sur toute

21. Les geais bleus manifestent un comportement agressif, qui inclut l'attaque des yeux, raison pour laquelle les Pueblo les associent à la guerre (Patrick Pérez, com. pers.). 
la Côte Nord-Ouest par leurs voix de sifflets (Boas 1897, p. 446; Swanton 1905, p. $156 ; 1908$, p. 436). Après une période de réclusion en forêt où le novice se transformait en incarnation du grand cannibale, son père lançait une opération pour le capturer et le ré-humaniser à l'aide de danses et de chants. C'est cette opération d'humanisation qui se déroulait dans la maison cérémonielle.

Cette maison partageait l'architecture d'une maison profane, dont elle se distinguait toutefois par l'ordre inversé des sièges (Boas 1897, p. 435) ${ }^{22}$ : la famille invitante (du père du novice) se repliait du côté de la porte, tandis que la place d'honneur était occupée par la plus haute société secrète, celle des phoques. Désignée en fonction du gibier principal, celle-ci incluait les cannibales, qui apparaissaient plutôt comme des oiseaux prédateurs ${ }^{23}$. Cette identité entre gibiers et prédateurs s'explique, comme dans les Plaines, par le fait que le rituel était pensé comme une chasse inversée: les animaux, proies des humains pendant l'été, devenaient leurs chasseurs pendant l'hiver (Reid 1979, p. 253). Face à ces prédateurs non-humains, les dirigeants du rituel (eux-mêmes d'anciens phoques) représentaient des passereaux, oiseaux terrestres et proies des aigles (Goldman 1975, p. 116, 191). Le novice étant transformé en cannibale pendant son séjour en forêt, la relation de départ entre lui et ses capteurs était donc celle entre un prédateur en haut et ses proies humaines en bas.

Ce lien se manifestait depuis la première apparition du novice. Chez les Kwagul, Heiltsuk et Haisla comme chez les Haida, il courait à travers le toit, grimpait sur le mât et attaquait les gens d'en bas, arrachant avec ses dents des morceaux de chair de leurs bras et leurs poitrines (Boas 1897, p. 527 et suiv.; Drucker 1940, p. 209; Olson 1940, p. 177; Murdock 1936, p. 6 et suiv.; Swanton 1905, p. 164 et suiv.) - du moins symboliquement; de fait, il coupait leur peau avec un couteau (Boas 1897, p. 440; Drucker 1940, p. 165), à l'instar des tortionnaires des Plaines. Cette homologie entre les morsures de la Côté Nord-Ouest et les «tortures » des Plaines (voir Reid 1981, p. 242) devient explicite chez les Nuu-chah-nulth, où les novices « punissaient » des offenseurs (désignés à l'avance) en perçant leurs bras avec des broches pour ensuite les tirer à la corde de harpon (Drucker 1951, p. 389).

Les danses et chants du rituel avaient pour but de ramener le novice de l'état cannibale à l'état humain, du haut vers le bas. Cette transformation commençait déjà avec son entrée lorsque, attiré par les chants des humains, il sautait dans la maison cérémonielle par le trou de la cheminée. Comme l'a montré Reid

22. Chez les Haida, cette inversion était accomplie en fermant la porte frontale et en ouvrant une nouvelle porte au fond (Swanton 1905, p. 165).

23. Le danseur cannibale était accompagné par des oiseaux anthropophages et portait parfois un masque de corbeau (Harkin 1997, p. 14; Boas 1897, p. 446). Selon le mythe d'origine haisla, le mangeur de corps n'était pas tant le danseur humain que l'esprit d'un oiseau qui le possédait, matérialisé par le sifflet (Olson 1940, p. 193). 
(1981, p. 230), ce saut reproduisait la chute mythique du grand cannibale dans la cavité du foyer que les humains avaient creusé en bas. Le prédateur descendait ainsi dans la maison cérémonielle comme un " oiseau de proie pris au piège " (ibid., p. 247); et le moyen pour le piéger consistait justement à s'offrir à ses morsures. Leurré par un homme qui servait d' « appât» puis par un cadavre qu'on lui offrait à manger (Boas 1897, p. 527 et suiv., 441), le novice devait être apaisé par des danses. Contrairement aux capteurs des Plaines qui transféraient la puissance surnaturelle aux danseurs en perçant leur chair, ceux de la Côte Nord-Ouest l'enlevaient au novice en dansant pour lui et en se faisant arracher la chair par lui.

L'association avec le cannibalisme n'est pas la seule affinité de ces danses avec l'épreuve de la danse du soleil. Les danses d'hiver étaient en effet exécutées sous le regard implacable des phoques. Si la moindre erreur était commise par le danseur, le novice retombait à l'état cannibale, les phoques attaquaient et mordaient alors le danseur, qui s'évanouissait pour être à son tour enlevé par l'esprit (Boas 1897, p. 433). Toujours susceptible de se transformer en torture, la danse était donc surtout une présentation au regard perçant des esprits, chargés, comme les geais bleus du plateau, d'une fonction de surveillance. Ce regard était un trait caractéristique du grand cannibale: selon une description légendaire, le mât qui le représentait était surmonté par l'aigle qui, assis sur la tête de l'ogre, guettait la viande humaine (Boas 1921, p. 856; Goldman 1975, p. 110). L'attaque par les yeux de l'oiseau entraînait l'attaque des yeux humains, nourriture favorite des esprits : le corbeau, assistant du grand cannibale, mangeait selon le mythe les yeux des victimes de son maitre (Boas 1897, p. 394). Chez les Tlingit, l'attaque du corbeau aux yeux humains se traduisait par une impossibilité de le fixer du regard: quiconque le regarderait l'œil nu se changerait en pierre (De Laguna 1972, p. 259) ${ }^{24}$. Les danses d'esprits de la Côte Nord-Ouest n'obligeaient pas les danseurs à fixer du regard le grand cannibale. Pourtant le rapport entre la danse et le regard se confirme par le négatif: comme l'a noté Lévi-Strauss (1979, p. 64, 129), le masque Dzonokwa, qui se distinguait pendant le rituel d'hiver par sa somnolence et son incapacité à danser (Boas 1897, p. 479 et suiv.), avait des yeux profondément enfoncés dans les orbites, c'est-à-dire éblouis. Si bien danser signifiait pouvoir affronter le regard des esprits, mal danser équivalait à ne plus rien voir. Tout en accentuant l'aspect passif (se présenter au regard) plutôt que l'aspect actif (fixer du regard), la danse des capteurs de la Côte Nord-Ouest était un combat de regards, autant que la danse des capturés des Plaines, dont elle fournissait la perspective inversée.

En dépit de cette inversion des relations entre capteurs et capturés, initiateurs et novices, leur modèle restait toujours celui d'un lien asymétrique entre promotions ou générations masculines. Ceci est explicite chez les Haida où les hôtes

24. Voir Lévi-Strauss 1979, p. 130. 
chargés d'humaniser les novices étaient les pères classificatoires de ceux-ci, membres de la moitié opposée (Swanton 1905, p. 162). Chez les Kwagul, où l'opposition entre groupes de parenté cédait en hiver à celle entre sociétés secrètes, les passereaux, anciens phoques, n'étaient pas considérés comme « pères » des phoques actuels, mais comme chez les Haida, le sponsor du rituel était le père du novice qui incarnait le cannibale. Toutefois, ce rapport initiatique entre « pères » et « fils » ne correspondait plus à un lien de filiation spirituelle. La transmission des danses d'esprits sur la Côte Nord-Ouest ne s'opérait pas en ligne masculine. Chez les Haisla et Heiltsuk, elles étaient héréditaires en ligne utérine (Olson 1940, p. 176; 1955, p. 337). Chez les Kwagul, il s'agissait, comme déjà évoqué, d'une transmission en ligne féminine implicite, le père recevant du père de sa femme le privilège de la danse que son fils allait exécuter. Bien qu'exclusivement réservé aux hommes, le lien central de la fête d'hiver était d'emblée médiatisé par les femmes.

Cette médiation prenait des formes multiples et complexes, dont l'ethnographie laisse encore beaucoup de questions ouvertes. Les femmes intervenaient en effet dans les deux sens, comme vecteurs de la transformation du novice en être surnaturel et de sa re-transformation en être humain. Bien que les deux interventions eussent lieu pendant le rite d'hiver, la première a été caractérisée à juste titre comme une « intrusion » de l'été (Rosman et Rubel 1990, p. 624), non seulement parce qu'elle mobilisait des groupes de parenté (institution « estivale » suspendue pendant la période des fêtes), mais aussi parce qu'il s'agissait d'un rite de séparation orienté vers l'extérieur. Pendant ce rite, le père du novice recevait le privilège de la danse (pour son fils) comme part d'une série de cadeaux par lesquels le groupe allié « rachetait » formellement son épouse, la mère du novice (Boas 1897, p. 421 et suiv.). Comme dans le coït rituel des Plaines, il s'agissait donc d'obtenir des puissances spirituelles en cédant l'épouse au transféreur - à la différence que le receveur ne les obtenait pas pour lui-même mais pour son fils, et que le transféreur n'était plus son « (grand-)père » mais son beau-père. Comme le « (grand-)père » des Plaines, le beau-père kwagul constituait toutefois, ainsi que l'a proposé Goldman (1975, p. 70), un équivalent de l'être surnaturel, vu que l'épouse kwagul était considérée comme un être d'en haut et quasiment non-humain ${ }^{25}$ - lors du rite de mariage il fallait la chercher au sommet du toit (Boas 1921, p. 1053 et suiv.). Contrairement au coït rituel des Plaines qui reproduisait le séjour mythique de la femme humaine parmi les astres, le « rachat » de la fiancée kwagul évoquait un retour de la femme céleste chez les siens; femme qui, au lieu d'attirer l'être surnaturel dans le domaine des humains, permettait à un être humain (son fils) d'être enlevé par les non-humains. La correspondance n'est certes pas parfaite, d'abord parce que la configuration $\mathrm{du}$ « rachat » implique non pas trois mais

25. Voir Lévi-Strauss 1979, p. 86 et suiv. sur la conception de la fiancée kwagul comme une ogresse apprivoisée, toujours susceptible de voler les enfants à la famille de son mari. 
quatre positions (la mère, le père, le beau-père et le fils), mais on voit que les deux rites étaient orientés en sens inverse. Si le coït rituel était une variante hivernale ou nocturne de la danse du soleil, le rachat de l'épouse était le volet estival de la fête d'hiver (voir Figure 8a).

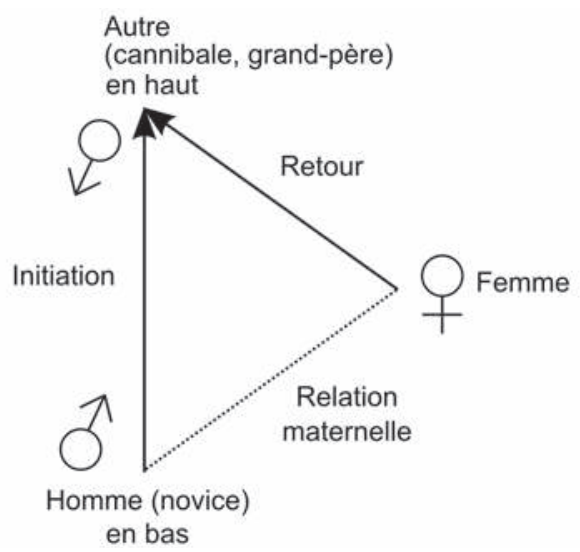

Fig. 8a - Triangle relationnel

(Côte Nord-Ouest, rachat de l'épouse).

La médiation féminine intervenait aussi au comble de la fête d'hiver, cette fois d'une façon tout à fait conforme à la logique du coït rituel: lorsque le novice, devenu grand cannibale entre-temps, retournait dans le monde humain, c'était une femme nue qui l'entraînait à l'intérieur de la maison, en marchant à reculons devant lui et en lui présentant un cadavre qu'elle portait sur ses mains (Boas 1897, p. 441 et suiv., 528). Toujours parente féminine du novice (ibid., p. 438), cette femme figurait la captive du grand cannibale, chargée de l'approvisionner en chair humaine (ibid., p. 394 et suiv.). Cette fonction nourricière la rapprochait d'une épouse, mais aussi de la captive humaine qui, selon le mythe d'origine de la fête d'hiver, avait aidé ses frères à tuer l'ogre et à lui dérober le rituel (Boas 1897, p. 396-400; Reid 1981, p. 137 et suiv.; Goldman 1975, p. 111). Si le « rachat » de l'épouse des humains, en la soustrayant à son mari, avait permis la transformation du novice en cannibale, la traîtrise de la captive du cannibale, en lui présentant un corps séduisant, médiatisait sa re-transformation en être humain.

Le corps féminin était en effet, comme dans les Plaines, le moyen ultime par lequel s'achevait le passage entre les mondes humain et non-humain. L'encens par lequel on purifiait le novice kwagul était la fumée de serviettes périodiques d'écorce de cèdre imbibées de sang menstruel (Reid 1981, p. 197). Toutefois, l'impact de ce sang était contraire à celui qu'il avait dans les Plaines. Dans la danse du soleil arapaho, boire l'« eau suave » mettait fin à la période de la soif 
et des saignements. Dans la fête d'hiver kwagul, la fumée purifiante ouvrait la période de réclusion où le novice ne pouvait boire l'eau qu'en petites quantités à travers un os d'aigle et était à tous égards traité comme une fille qui a ses premières règles (Boas 1897, p. 538; Goldman 1975, p. 96). Si le novice des Plaines, saignant et suspendu au mât comme la première femme menstruée, se transformait à la fin en buveur de sang menstruel, le cannibale de la Côte Nord-Ouest, après avoir fait couler le sang humain, finissait assoiffé et enfermé comme une fille recluse ${ }^{26}$.

Au détail près que la médiation féminine passait sur la Côte Nord-Ouest par l'échange matrimonial et non pas, comme dans les Plaines, par l'échange d'épouses, le triangle relationnel qui caractérisait le rituel d'hiver correspond donc au triangle de la danse du soleil dont l'axe central a été mis sens dessus dessous (voir Figure 8b).

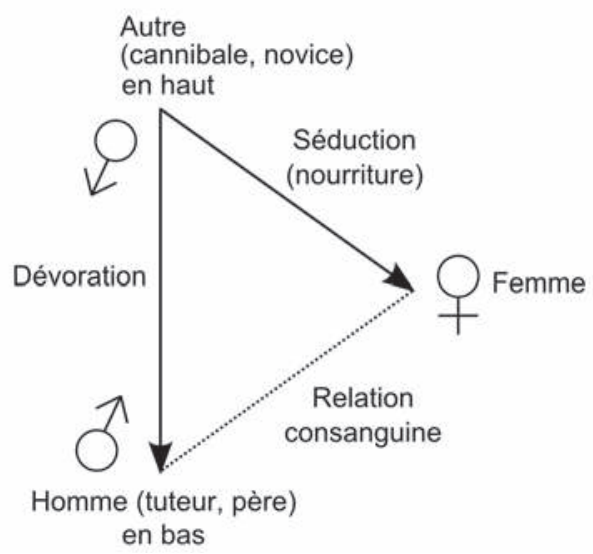

Fig. $8 \mathrm{~b}$ - Triangle relationnel

(Côte Nord-Ouest, capture du cannibale).

$$
* *
$$

Des Plaines à la Côte Nord-Ouest en passant par le Plateau, les grands rituels saisonniers des sociétés amérindiennes modélisaient le même espace relationnel selon des perspectives variables. Dans tous les cas, l'axe central de cet espace était constitué par un antagonisme entre un prédateur non humain en haut et son gibier humain en bas, et le rituel visait à inverser leurs positions. Dans les Plaines, il s'agissait de transférer la puissance surnaturelle aux humains en bas pour les

26. Le parallélisme devient explicite chez les Nuu-chah-nulth, qui admettaient des filles comme novices lors du rituel d'hiver, généralement au moment des premières règles (Ernst 1952, p. 12; Boas 1891, p. 600). 
transformer en prédateurs; sur la Côte Nord-Ouest, il s'agissait de l'enlever aux non-humains en haut pour les ramener à l'état humain. Conformément aux techniques estivale et hivernale de la chasse, l'action rituelle consistait dans un cas à envoyer les humains vers l'Autre, dans l'autre cas à attirer l'Autre vers les humains. Dans les deux sens, le passage était mis en œuvre comme une transformation de perspective - d'où l'importance cruciale de l'action par et sur le regard. L'axe central du rituel reliait les yeux perçants du cannibale céleste et les yeux dévorés des humains dans un rapport de transmission qui, selon le cas, rendait les humains clairvoyants ou éblouissait le cannibale.

Localisé au centre de l'espace social au comble de sa concentration périodique, cet axe constituait un lien masculin, sans pour autant exclure les femmes, bien au contraire. Dans les versions « hivernales » du rite, la femme médiatisait le lien central, soit en apportant la puissance surnaturelle à son mari humain dans les Plaines, soit en l'enlevant à son mari non humain sur la Côte Nord-Ouest. Même les versions « estivales » et non médiatisées impliquaient foncièrement la référence à la femme, que ce soit comme vierge invincible dans les Plaines, ou comme épouse rachetée sur la Côte Nord-Ouest. Dans tous les cas, le mouvement envisagé était un rapprochement entre la femme et l'Autre, considéré, dans une optique « hivernale », comme venue de l'Autre, ou dans une optique « estivale », comme éloignement de la femme. Tout en alternant selon une logique saisonnière, les deux optiques se retrouvaient au sein même des rituels centraux. Nous pouvons donc réunir les schémas précédents (Figures 2, 7 et 8) en un schéma synthétique (Figure 9, page suivante) ${ }^{27}$ afin d'esquisser, non pas un super-rituel parfaitement cohérent et symétrique, mais un système de coordonnées aidant à comprendre les nombreuses résonances entre les rituels qui s'y inscrivent.

Ce modèle nous invite à repenser la différence des genres en tant que modes de la relation à l'Autre. Dans une précédente étude consacrée à l'espace social amazonien (Hamberger 2012), nous avons proposé de distinguer ces deux modes selon leur caractère immédiat ou médiatisé. Dans la conception des sociétés amazoniennes, le lien entre les hommes et l'Autre se fondait sur la coupure d'une relation primordiale que cet Autre entretenait avec les femmes - relation qui représentait, au sud comme au nord du continent américain, un canal primordial de transmissions spirituelles, origine ou but de la culture. Reléguant la femme à la périphérie et l'enfermant à l'intérieur, l'homme était désormais censé médiatiser les rapports de la femme avec l'Autre - d'où sa position au centre de l'espace social, tourné vers l'extérieur. Cette formule n'était pas étrangère aux sociétés de l'Amérique du Nord, comme le montrent des institutions telles que le rite cheyenne du renouvellement des flèches sacrées

27. Je remercie le lecteur anonyme du $J S A$ qui m’a suggéré cette représentation synthétique. 


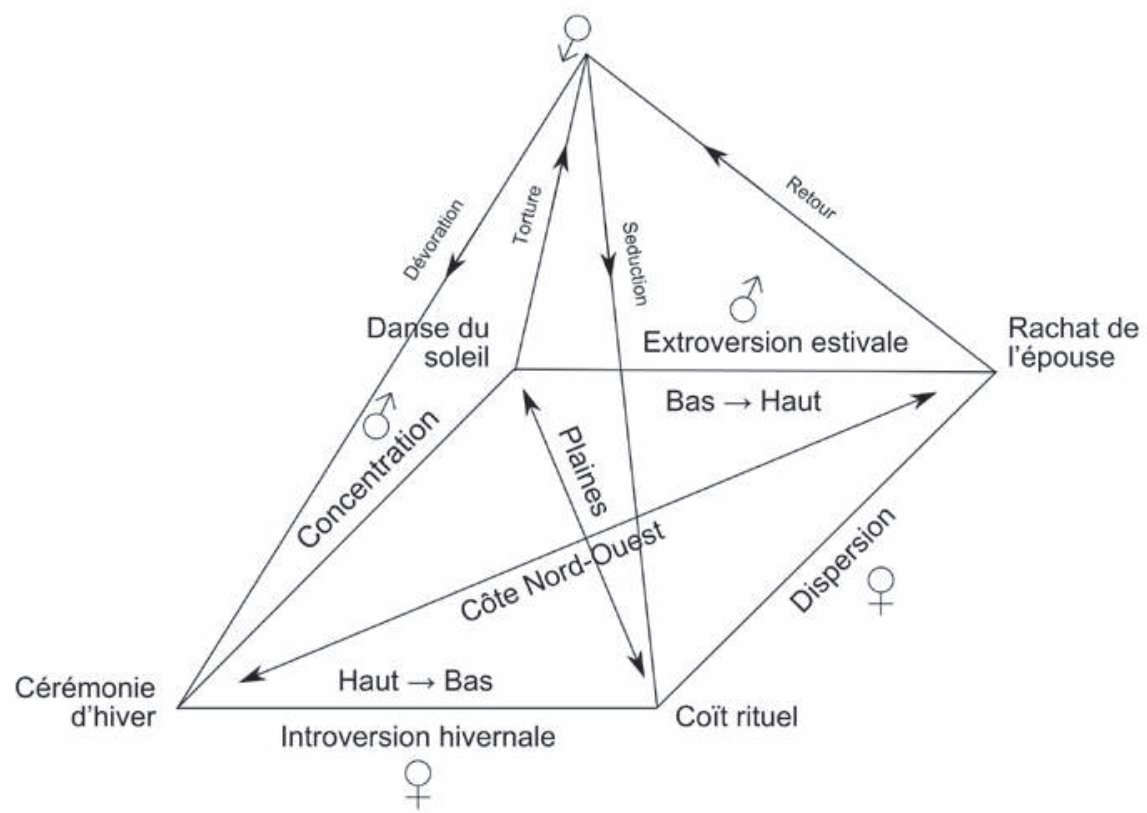

Fig. 9 - Schéma synthétique de l'espace relationnel.

(qui excluait les femmes, comme les rites des flûtes sacrées d'Amérique du Sud) ${ }^{28}$ ou les rites des scalps (destinés aux épouses, comme les têtes réduites d'Amérique du Sud ${ }^{29}$. Elle n'y constituait pourtant qu'un aspect partiel d'une logique relationnelle que l'on pouvait aussi mobiliser en sens inverse, à l'instar des institutions qui, au lieu de couper ou de médiatiser le lien entre la femme et l'Autre, faisaient au contraire appel à la femme pour médiatiser le lien entre l'homme et l'Autre. Ce dernier aspect prédomine dans les rituels discutés tout au long de cet article.

L'étude des sociétés nord-amérindiennes fait ainsi apparaître les contours d'un modèle plus général qui, au lieu de distinguer les genres masculin et féminin par la relation directe ou médiatisée à l'Autre, les comprend plutôt comme deux modes différents pour établir la relation à l'Autre, tantôt en le poursuivant, tantôt en l'attirant, accentuant ainsi en alternance ses deux faces d'ennemi et d'amant. Cette alternance entre extroversion et introversion pouvait s'accorder au rythme de la concentration et de la dispersion, par lequel l'extérieur se transformait périodiquement en intérieur et inversement. Mais il n'y avait à cet égard aucun

28. Voir Hoebel 1960, p. 6-11. Comme le soleil (qui était aux solstices au moment du rituel), les flèches aussi émettaient une lumière éblouissante et étaient difficiles à regarder.

29. Voir Lévi-Strauss 1968, p. 328 et suiv. 
déterminisme rigide. La forme dynamique d'un modèle topologique qui calquait le centre sur la périphérie et comprenait la société toute entière sous la forme d'une maison favorisait le passage entre ces deux formes d'antagonisme que sont la guerre et la séduction, et plaçait, au comble même de la concentration masculine, la femme dans l'origine de l'espace social - origine qui n'était de fait pas tant un lieu qu'un moment, dont chaque année éprouvait le retour. *

* Manuscrit reçu en août 2015, accepté pour publication en février 2016.

\section{Références citées}

BARNES Robert Harrison

1984, Two Crows denies it: a history of controversy in Omaha sociology, University of Nebraska Press, Lincoln.

Black Elk (Hehaka Sapa)

1953, The sacred pipe, Black Elk's account of the seven rites of the Oglala Sioux, Joseph Epes Brown (éd.), University of Oklahoma Press, Norman.

BoAs Franz

1891, Second general report on the Indians of British Columbia. II. The Nootka, John Murray, London.

1897, The social organization and the secret societies of the Kwakiutl Indians, Reports of the United States National Museum, Government Printing Office, Washington (DC).

1909, The Kwakiutl of Vancouver Island, Brill, Leiden (Memoirs of the American Museum of Natural History, vol. 8, part 2)/Stechert (Publications of the Jesup North Pacific Expedition, vol. 5, part 2), New York.

1920, "The social organization of the Kwakiutl », American anthropologist, 22, p. 111-126.

1930, The religion of the Kwakiutl Indians, Columbia University Press (Columbia University Contributions in Anthropology, 10), New York.

1934, Geographical names of the Kwakiutl Indians, Columbia University Press (Columbia University Contributions in Anthropology, 20), New York.

1966, Kwakiutl ethnography, Helen Codere (éd.), The University of Chicago Press, Chicago.

BoAs Franz et George T. HunT

1905, Kwakiutl texts, Brill, Leiden/Stechert, New York.

1921, «Ethnology of the Kwakiutl», in Thirty-fifth annual report of the Bureau of American Ethnology to the Secretary of the Smithsonian Institution, 1913-1914, Government Printing Office, Washington (DC), p. 41-1581.

Bowers Alfred W.

1950, Mandan social and ceremonial organization, University of Idaho Press, Moscow.

1965, Hidatsa social and ceremonial organization, Government Printing Office, Washington (DC).

Bushnell David I.

1922, Villages of the Algonquian, Siouan, and Caddoan tribes west of the Mississippi, Government Printing Office, Washington (DC). 
CATLIN George

1867, O-Kee-Pa: a religious ceremony and other customs of the Mandans, Yale University Press, London/New Haven.

Chamberlain Von Del

1982, When stars came down to earth: cosmology of the Skidi Pawnee Indians of North America, Ballena Press, Los Altos (CA).

De Laguna Frederica

1972, Under Mount Saint Elias: the history and culture of the Yakutat Tlingit, Smithsonian Institution Press, Washington (DC).

Deloria Ella

1929, « The sun dance of the Oglala Sioux », Journal of American Folk-Lore, 42 (166), p. 354-413.

DeMallie Raymond J.

1983, « Male and female in traditional Lakota culture », in Patricia Albers et Beatrice Medicine, The hidden half, University Press of America, New York, p. 237-265.

DÉSvEAux Emmanuel

1997, « Parenté, rituel, organisation sociale: le cas des Sioux », Journal de la société des américanistes, 83, p. 111-140.

2001, Quadratura americana: essai d'anthropologie lévi-straussienne, Georg éditeur, Genève.

2007 [2000], « Guerre et mariage, confréries et clans, aperçu sociologique et rituel », in Spectres de l'anthropologie. Suite nord-américaine, Aux lieux de l'être, Montreuil, p. 247-271.

DoRSEY George A.

1903, The Arapaho sun dance: the ceremony of the Offerings lodge, [s. n.] (Field Columbian Museum, 75; Anthropological series, 4), Chicago.

1905a, The Ponca sun dance, [s. n.] (Field Columbian Museum, 102; Anthropological series, vol. 7, $\mathrm{n}^{\circ}$ 2), Chicago, p. 51-88.

1905b, The Cheyenne. II. The sun dance, Field Columbian Museum (Field Columbian Museum, 103; Anthropological series, vol. 9, n 2), Chicago.

1906, « The Skidi rite of human sacrifice », Proceedings of the International Congress of Americanists, 15 (2), p. 65-70.

Dorsey James Owen

1894, « A study of Siouan cults », in Eleventh annual report of the Bureau of Ethnology to the Secretary of the Smithsonian Institution, 1889-1890, Government Printing Office, Washington (DC).

DRUCKer Philip

1940, « Kwakiutl dancing societies », Anthropological records, 2 (6), p. 201-230.

1951, The northern and central Nootkan tribes, Government Printing Office, Washington (DC).

Emmons George Thornton

1991, The Tlingit Indians, University of Washington Press, Seattle/American Museum of Natural History, New York.

ERnst Alice Henson

1952, The Wolf ritual of the northwest coast, University of Oregon, Eugene. 
Ewers John Canfield

1958, The Blackfeet: raiders of the northwestern Plains, University of Oklahoma Press, Norman.

FORTUNE Reo Franklin

1932, Omaha secret societies, Columbia University Press, New York.

GODDARD Pliny Earl

1919, « Notes on the sun dance of the Cree in Alberta », Anthropological papers of the American Museum of Natural History, 16 (4), p. 295-310.

GoLDMAN Irving

1975, The mouth of heaven: an introduction to Kwakiutl religious thought, John Wiley and Sons, New York/London/Sydney.

GrinNELL George Bird

1923, The Cheyenne Indians, their history and ways of life, Yale University Press, New Haven, 2 vol.

HAMBERGER Klaus

2012, « Le modèle topologique des sociétés amazoniennes », Journal de la société des américanistes, 98 (2), p. 199-232.

HARKIN Michael E.

1997, The Heiltsuks: dialogues of culture and history on the Northwest Coast, University of Nebraska Press, Lincoln.

HASSRICK Royal B.

1964, The Sioux: life and customs of a warrior society, in collaboration with Dorothy Maxwell and Cile M. Bach, University of Oklahoma Press, Norman.

HiLger Inez M.

1952, Arapaho child life and its cultural background, Government Printing Office, Washington.

Hoebel E. Adamson

1960, The Cheyennes: Indians of the Great Plains, Holt, Rinehart \& Winston, New York.

KeHOE Alice Beck

1995, « Blackfoot persons », in Lilian A. Ackerman et Laure F. Klein (ed.), Women and power in native North America, University of Oklahoma Press, Norman, p. 113-125.

KROEBER Alfred L.

1908, «Ethnology of the Gros Ventre », Anthropological papers of the American Museum of National History, 1 (4), p. 145-281.

LÉVI-STRAuss Claude

1958 [1956], « Structure et dialectique », in Anthropologie structurale, Plon, Paris, p. 266-275.

1968, L'Origine des manières de table (Mythologiques III), Plon, Paris.

1971, L'Homme nu (Mythologiques IV), Plon, Paris.

1973 [1971], « Rapports de symétrie entre rites et mythes de peuples voisins », Anthropologie structurale deux, Plon, Paris, p. 281-300.

1979, La voie des masques, édition revue, augmentée et rallongée de trois excursions, Flammarion, Paris. 
LINTON Ralph

1922, The sacrifice to the morning star by the Skidi Pawnee, Field Museum of Natural History (Anthropology leaflet, 6), Chicago, p. 1-181.

1935, "The Comanche sun dance », American anthropologist, New Series, 37 (3), Part 1, p. 420-428.

LOwIE Robert H.

1913, « Societies of the Crow, Hidatsa, and Mandan Indians », Anthropological papers of the American Museum of Natural History, 11 (3), p. 219-358.

1915, " The sun dance of the Crow Indians ", Anthropological papers of the American Museum of Natural History, 16 (1), p. 1-50.

1916, " Plains Indians age-societies: historical and comparative summary ", Anthropological papers of the American Museum of Natural History, 11 (13), p. 877-984.

1919, « Sun dance of the Shoshoni, Ute, and Hidatsa », Anthropological papers of the American Museum of Natural History, 16 (5), p. 387-431.

Mandelbaum David G.

1940, « The Plains Cree. An ethnographic, historical and comparative study », Anthropological papers of the American Museum of Natural History, 37 (2).

MAUZÉ Marie

1989, « L'organisation politique des kwagul méridionaux au XIX ${ }^{\mathrm{e}}$ siècle », Journal de la société des américanistes, 75, p. 173-192.

Maximilian zu Wied-Neuwied

1843, Travels in the interior of North America, translated from the German by H. Evans Lloyd, Ackermann \& Co., London.

McLEAN John

1888, « The Blackfoot sun dance », Proceedings of the Canadian Institute, 6 ( $3^{\text {rd }}$ series), p. 231-237.

Moore John H.

1974, « Cheyenne political history, 1820-1894 », Ethnohistory, 21 (4), p. 329-359.

MÜLLER Werner

1955, Weltbild und Kult der Kwakiutl-Indianer, Franz Steiner, Wiesbaden.

Murdock George P.

1936, Rank and Potlatch among the Haida, Yale University Press, New Haven.

Murie James R.

1989, Ceremonies of the Pawnee, Smithsonian Institution Press, Washington, 2 vol.

OBerg Kalvero

1973, The social economy of the Tlingit Indians, University of Washington Press, Seattle/London.

Olson Ronald L.

1940, «The social organization of the Haisla of British Columbia », Anthropological records, 2 (5), p. 169-200.

1955, « Notes on the Bella Bella Kwakiutl », Anthropological records, 14 (5), p. 319-348.

1967, Social structure and social life of the Tlingit in Alaska, University of California Press, Berkeley. 
RAY Verne F.

1933, The Sanpoil and Nespelem: Salishan peoples of Northeastern Washington, University of Washington Press, Seattle/Washington.

1937, « The Bluejay character in the Plateau Spirit Dance », American anthropologist, 39 (4), p. 593-601.

1939, Cultural relations in the plateau of northwestern America, The Southwest Museum (Publications of the Frederick Webb Hodge Anniversary Publication Fund, 3), Los Angeles.

REID Martine-Jeanne

1981, La cérémonie Hamatsa des Kwagul. Approche structuraliste des rapports mytherituel, thèse de doctorat, University of British Columbia.

ReID Susan

1979, « The Kwakiutl Man Eater », Anthropologica, 21 (2), p. 247-276.

Rosman Abraham et Paula G. Rubel

1971, Feasting with mine enemy: rank and exchange among northwest coast societies, Columbia University Press, New York.

1990, « Structural patterning in Kwakiutl art and ritual », Man, 25, p. 620-639.

SPIER Leslie

1921, "The sun dance of the Plains Indians: its development and diffusion », Anthropological papers of the American Museum of Natural History, 16 (7), p. 451-527.

SwANTON John Reed

1905, Contributions to the ethnology of the Haida, Brill, Leiden (Memoirs of the American Museum of Natural History, vol. VIII, part 1)/Stechert, New York.

1908, Social condition, beliefs and linguistic relationships of the Tlingit Indians, $26^{\text {th }}$ Annual report of the Bureau of American Ethnology, Government Printing Office, Washington (DC).

THURMAn Melburn D.

1983, « The timing of the Skidi-Pawnee morning star sacrifice », Ethnohistory, 30 (1/4), p. 155-163.

TuRNEY-High Harry Holbert

1933, « The Bluejay dance », American anthropologist, 35 (1), p. 103-107.

1937, The Flathead Indians of Montana, American Anthropological Association (Memoirs of the American Anthropological Association, 48), Menasha (réimprimé: Kraus Reprint, New York, 1969).

1941, Ethnography of the Kutenai, American Anthropological Association (Memoirs of the American Anthropological Association, 56), Menasha (réimprimé: Kraus Reprint, New York, 1971).

WALKER James R.

1917, « The sun dance and other ceremonies of the Oglala division of the Teton Dakota », Anthropological papers of the American Museum of Natural History, 16 (2), p. 51-221.

1991, Lakota belief and ritual, Raymond J. DeMallie et Elaine A. Jahner (éd.), University of Nebraska Press, Lincoln/London. 
Klaus Hamberger

\section{WeltFish Gene}

1965, The lost universe, Basic Books, New York/London.

WiLl George Francis et Herbert Joseph SpINDEN

1906, The Mandans. A study of their culture, archeology and language, [s. n.] (Papers of the Peabody Museum of American archaeology and ethnology, vol. 3, part 4), Cambridge (MA).

WisSLER Clark

1918, "The sun dance of the Blackfoot Indians », Anthropological papers of the American Museum of Natural History, 16 (3), p. 223-270. 